\title{
The effect of voids shape on hypervelocity cylindrical cavity expansion and shock waves formation in transversely isotropic porous materials
}

\author{
T. dos Santos ${ }^{\mathrm{a}, *}$, J. C. Nieto-Fuentes ${ }^{\mathrm{b}}$, N. Hosseini ${ }^{\mathrm{b}}$, J. A. Rodríguez-Martínez ${ }^{\mathrm{b}}$ \\ ${ }^{a}$ Departamento de Engenharia Mecânica, Universidade Federal de Santa Maria, Av. Roraima, 1000, Prédio 7, Santa Maria, RS, \\ 97105-900, Brazil. \\ ${ }^{b}$ Department of Continuum Mechanics and Structural Analysis, University Carlos III of Madrid, Avda. de la Universidad, 30, 28911 \\ Leganés, Madrid, Spain
}

\begin{abstract}
This paper investigates the steady-state dynamic radial expansion of a pressurized circular cylindrical cavity in an infinite porous medium modeled with the constitutive framework developed by Monchiet et al. (2008), which considers the material to display a periodic porous microstructure with spheroidal voids and matrix described by the orthotropic yield criterion of Hill (1948). For that purpose, we have extended the formulation of dos Santos et al. (2019b) to consider oblate and prolate voids, which allows to assess the role of the initial voids shape on the elastoplastic-anisotropic fields that develop near the cavity. The theoretical development follows the cavity expansion formalism of Cohen and Durban (2013) and employs the artificial viscosity approach of Lew et al. (2001) to avoid singularities in the field variables due to the formation of plastic shock waves. The main outcome of this work is a relationship between the critical cavity expansion velocity for which plastic shocks emerge and the initial aspect ratio of the spheroidal voids. The results show that the formation of shocks is delayed for oblate voids, in comparison with spherical and prolate voids. These findings have been substantiated for different anisotropic behaviors and initial void volume fractions.
\end{abstract}

Keywords: Cylindrical cavity expansion, Matrix anisotropy, Ductile materials, Spheroidal voids, Plastic shocks

\section{Introduction}

\footnotetext{
${ }^{*}$ Corresponding author. E-mail address: tiago.santos@ufsm.br
} 
2017; du Plessis et al., 2020). In particular, when it comes to impact applications, the interplay between material porosity, plastic anisotropy and inertia on the perforation/penetration mechanics of printed structures needs to be assessed. In the words of Costas et al. (2021), additive manufacturing has a large unrealised potential in the field of ballistic protection, with the largest drawback being the uncertainty surrounding the mechanical properties of additive manufactured materials under impulsive loading.

Currently, there are only a few experimental studies concerning the perforation/penetration of printed structures. For instance, Kristoffersen et al. (2020) investigated the ballistic perforation resistance of additive manufactured aluminum plates employing both experimental and numerical analysis. The authors observed negligible difference in ballistic performance between the additive manufactured and die-cast materials. As an important outcome, they concluded that a printed material may have similar or even better ballistic properties than a traditionally-manufactured equivalent material. Moreover, Costas et al. (2021) addressed the ballistic properties of a selective laser melting additive manufactured maraging high-strength steel and demonstrated the potential of this material in protective applications, which could be even better had the presence of large voids and particles been reduced.

In absence of experimental data, theoretical and numerical analysis are essential to advance in the knowledge of the mechanisms that control the perforation/penetration of printed materials. In this context, the cavity expansion theory has been accepted as a simple and fairly accurate approach to understand the underlying mechanics of penetration/perforation problems (Forrestal et al., 1990, 1991; Durban and Masri, 2004; Masri and Durban, 2005). The key advantage of the cavity expansion theories is to provide simple expressions to predict the pressure-velocity relationship during penetration and also to estimate the mechanical fields close to the cavity. For example, the cylindrical cavity analogy was used by Forrestal et al. (1990) and Forrestal and Warren (2009) to obtain closed-form perforation equations for the ballistic limit and residual velocities for conical and ogival nose rigid projectiles penetrating aluminum targets. Masri and Durban (2009) studied deep penetration problems using the dynamic cylindrical cavitation model, and developed a practical procedure for estimating penetration the depth of rigid axisymmetric projectiles, using different plasticity theories to model the mechanical behavior of the targets, including von Mises and Tresca. Following the formalism of Cohen and Durban (2013), the model of Masri and Durban (2009) was extended by dos Santos et al. (2019b) in order to study cylindrical cavity expansion in orthotropic porous materials modeled with the constitutive framework of Benzerga and Besson (2001). The authors investigated the influence of key material features, such as plastic anisotropy, initial porosity and strain hardening, on the plastic shock wave emergence and on the elastoplastic fields that develop near the cavity. Among other conclusions, it was found that the formation and location of shock waves is practically independent of the anisotropic nature of the matrix material and the initial porosity was shown to promote the emergence of shocks for lower cavitation velocities.

Although the framework developed by dos Santos et al. (2019b) accounted for plastic anisotropy, the adopted 
porous plasticity model (Benzerga and Besson, 2001) was based on the assumption of persisting spherical voids. However, additive manufactured materials also contain non-spherical pores (de Terris et al., 2019; Sola and Nouri, 2019), and the shape of the voids affects the response of porous ductile materials (Gologanu et al., 1993, 1994, 1997), specially when the matrix material is anisotropic (Monchiet et al., 2008; Keralavarma and Benzerga, 2010; Morin et al., 2015; Tvergaard and Nyvang Legarth, 2019). Specific micromechanics-based models have been proposed in order to consider the interplay between anisotropy and voids shape in the mechanical response of the material. For example, Monchiet et al. (2008) extended the Gologanu-Leblond-Deveaux's model (Gologanu et al., 1993, 1994), assuming spheroidal (prolate/oblate) voids embedded in a Hill (1948) matrix material, so that the authors obtained a closed-form macroscopic yield function depending on the void volume fraction, the void shape, and the directionality of the plastic properties. Moreover, Keralavarma and Benzerga (2010) extended the Gologanu-Leblond-Perrin-Deveaux's model (Gologanu et al., 1997), which employed different kinematic conditions in comparison to Gologanu et al. $(1993,1994)$, and derived plastic constitutive relations for anisotropic porous materials containing spheroidal voids in an plastically anisotropic matrix (Hill, 1948). Unlike Monchiet et al. (2008), the authors also proposed evolution equations for the void volume fraction, the void shape, and the orientation. The model of Keralavarma and Benzerga (2010) was assessed in a subsequent work using unit cell finite element simulations (Keralavarma and Benzerga, 2015). This constitutive model was shown to provide a robust framework to be used in large-scale simulations of ductile fracture accounting for the combined effects of stress triaxiality, void shape, void orientation, matrix orthotropy, and loading path (see Kweon et al. (2016)). Morin (2015) compared the models of Monchiet et al. (2008) and Keralavarma and Benzerga (2010) against unit cell finite element calculations. The results demonstrated that, when spheroidal voids are considered, both models provide more precise predictions for the effective yield loci, in comparison with porous plasticity models based on the assumption of persisting spherical voids (Gurson, 1977; Benzerga and Besson, 2001). Moreover, Morin et al. (2015) extended Madou-Leblond's proposal (Madou and Leblond, 2012a,b) and developed a criterion for general ellipsoidal cavities embedded in a plastically anisotropic material also obeying Hill's criterion. In contrast to the works of Monchiet et al. (2008) and Keralavarma and Benzerga (2010), the study of Morin et al. (2015) was not restricted to spheroidal voids, so that ellipsoidal voids with arbitrary shapes were considered at the expense of a more complex formulation.

In this work, we extend the dynamic cavity expansion model of dos Santos et al. (2019b) in order to assess the coupled effects of voids shape and plastic anisotropy on the elastoplastic fields that develop near the cavity, and on the formation of plastic shocks at high cavitation velocities. The theoretical development follows the cavity expansion framework of Cohen and Durban (2013) using the artificial viscosity method (Lew et al., 2001) in order to avoid singularities related to the emergence plastic shock waves. The main novelty of this work is to model the material behavior with the yield criterion of Monchiet et al. (2008), which has a simpler formulation compared with the model of Keralavarma and Benzerga (2010), being especially suited for its implementation in 
where $w=\frac{a_{1}}{b_{1}}$. of plastic shocks. et al., 1997):

analytical models, with the material microstructure, void volume fraction and void shape, evolving according to the constitutive equations of Gologanu et al. $(1993,1994)$. As a main result, this work shows the influence of voids initial aspect ratio and matrix anisotropy on the critical cavity expansion velocity which leads to the formation

\section{Constitutive framework}

The mechanical behavior of the material is described using the constitutive model for anisotropic porous metals developed by Monchiet et al. (2008), who extended the formulation of Gologanu et al. $(1993,1994)$ to consider materials with matrix described by the orthotropic yield criterion of Hill (1948).

The model of Monchiet et al. (2008) assumes that the material displays a periodic porous microstructure that can be approximated by an array of representative volume elements idealized as spheroidal unit-cells which contain a confocal spheroidal void, see Fig. 1. The semi-axes of the spheroidal void and the spheroidal unit-cell are $\left(a_{1}, b_{1}\right)$ and $\left(a_{2}, b_{2}\right)$, respectively, and the corresponding eccentricities are defined as:

$$
\begin{aligned}
e_{1} & =\frac{c}{a_{1}} \text { and } e_{2}=\frac{c}{a_{2}} \text { (prolate) } \\
e_{1} & =\frac{c}{b_{1}} \text { and } e_{2}=\frac{c}{b_{2}} \text { (oblate) }
\end{aligned}
$$

where $c=\sqrt{\left|a_{1}^{2}-b_{1}^{2}\right|}=\sqrt{\left|a_{2}^{2}-b_{2}^{2}\right|}$ is the focal distance.

Moreover, the void volume fraction, $f$, and the void shape parameter, $S$, can be expressed as:

$$
\begin{gathered}
f=\frac{a_{1} b_{1}^{2}}{a_{2} b_{2}^{2}} \\
S=\ln \left(\frac{a_{1}}{b_{1}}\right)
\end{gathered}
$$

so that $S>0$ stands for a prolate void and $S<0$ for an oblate void. Note that given the void volume fraction $f$ and the void shape parameter $S$, the eccentricities can also be calculated using the following expressions (Gologanu

$$
e_{1}^{2}=\left\{\begin{array}{ll}
1-\frac{1}{w^{2}} & \text { (prolate) } \\
1-w^{2} & \text { (oblate) }
\end{array} \quad \text { and } \quad \frac{e_{2}^{3}}{\left(1-e_{2}^{2}\right)^{n}}=f \frac{e_{1}^{3}}{\left(1-e_{1}^{2}\right)^{n}} \quad \text { with } \quad n= \begin{cases}1 & \text { (prolate) } \\
1 / 2 & \text { (oblate) }\end{cases}\right.
$$




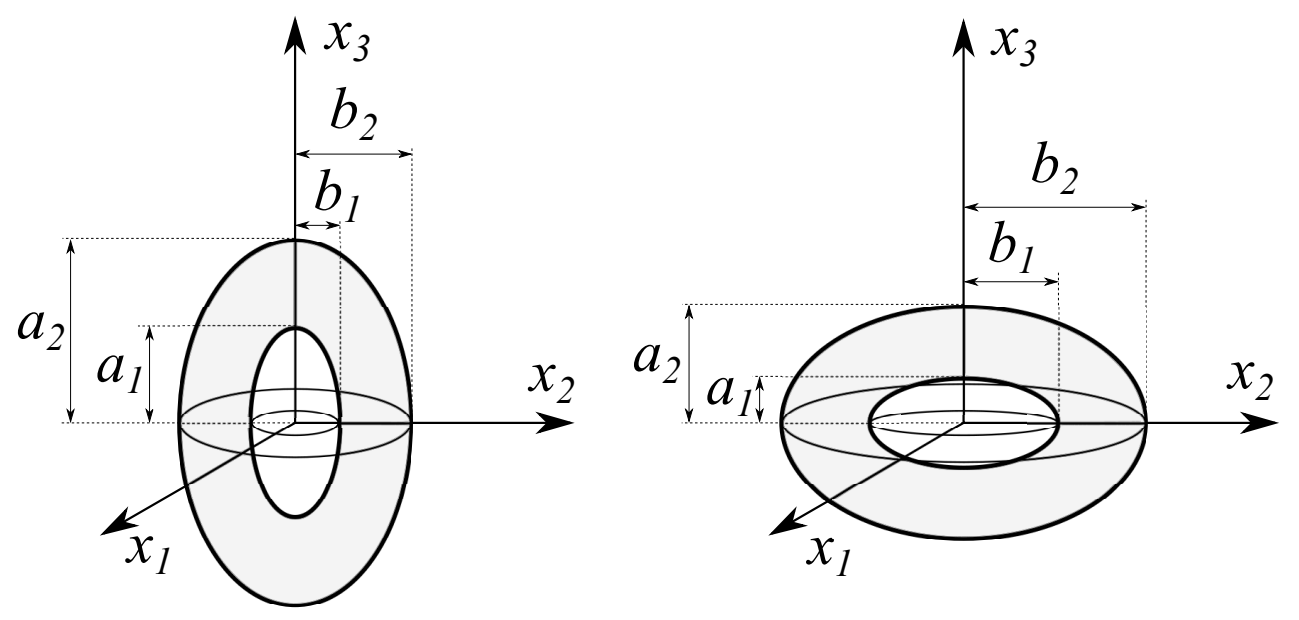

Figure 1: Spheroidal unit-cell containing a confocal spheroidal void referred to a Cartesian coordinate system $\left(x_{1}, x_{2}, x_{3}\right)$, with $x_{3}$ as the symmetry axis: (a) prolate void and (b) oblate void.

\subsection{General equations for elastic/plastic materials}

The rate of deformation tensor $\boldsymbol{d}$ is additively decomposed into an elastic part, $\boldsymbol{d}^{e}$, and a plastic part, $\boldsymbol{d}^{p}$, as follows:

$$
\boldsymbol{d}=\boldsymbol{d}^{e}+\boldsymbol{d}^{p}
$$

The elastic rate of deformation tensor is related to the Cauchy stress tensor, $\boldsymbol{\sigma}$, by the following hypo-elastic law:

$$
\stackrel{\nabla}{\sigma}=C: d^{e}
$$

with $\stackrel{\nabla}{()}$ being an objective derivative and $C$ the isotropic elastic tensor:

$$
\boldsymbol{C}=\frac{E}{1+\nu} \boldsymbol{I}^{\prime}+\frac{E}{3(1-2 \nu)} \mathbf{1} \otimes \mathbf{1}
$$

where $E$ is the Young's modulus, $\nu$ is the Poisson's ratio, $\mathbf{1}$ is the unit second-order tensor and $\boldsymbol{I}^{\prime}$ is the unit deviatoric fourth-order tensor.

The plastic rate of deformation tensor is related to the Cauchy stress tensor by an associated flow rule:

$$
\boldsymbol{d}^{p}=\dot{\lambda} \frac{\partial \Phi}{\partial \boldsymbol{\sigma}}
$$

where $\Phi$ is the flow potential, see Eq. (12), and $\dot{\lambda}$ is a non-negative rate of plastic multiplier satisfying the loading/unloading Kuhn-Tucker conditions: 


$$
\dot{\lambda} \geq 0 ; \quad \Phi \leq 0 ; \quad \dot{\lambda} \Phi=0
$$

and the consistency condition:

$$
\dot{\lambda} \dot{\Phi}=0
$$

where the superimposed dot denotes differentiation with respect to time.

\subsection{Monchiet et al. (2008) yield criterion}

Using a limit analysis approach, Monchiet et al. (2008) derived the following flow potential:

$$
\Phi=\left(\frac{\bar{\sigma}_{e}}{\sigma_{y}}\right)^{2}+2(1+g)(f+g) \cosh \left(\frac{\kappa \sigma_{p}}{\sigma_{y}}\right)-(1+g)^{2}-(f+g)^{2}
$$

where $g$ is a void shape parameter, see Eq. (23), $\kappa$ is a function that depends on the void shape, the void volume fraction and the anisotropy of the material, see Eq. (20), $\sigma_{p}$ is a scalar stress measure, see Eq. (16), $\bar{\sigma}_{e}$ is the generalized effective stress, see Eq. (15), and $\sigma_{y}$ is the yield strength of the matrix material defined as follows:

$$
\sigma_{y}=\sigma_{y 0}\left(1+\frac{\bar{\varepsilon}^{p}}{\varepsilon_{0}}\right)^{n}
$$

where $\sigma_{y 0}$ is the initial yield strength, $n$ is the strain hardening exponent and $\varepsilon_{0}$ is the reference strain. Moreover, $\bar{\varepsilon}^{p}=\int_{0}^{t} \dot{\bar{\varepsilon}}^{p}(\tau) d \tau$ is the effective plastic strain of the matrix material and $\dot{\bar{\varepsilon}}^{p}$ is the effective plastic strain rate, the latter being the work conjugate of the yield strength of the matrix material. The effective plastic strain rate is related to the plastic rate of deformation tensor assuming that the rate of macroscopic plastic work is equal to the rate of effective plastic work in the matrix material:

$$
\boldsymbol{\sigma}: \boldsymbol{d}^{p}=(1-f) \sigma_{y} \dot{\bar{\varepsilon}}^{p}
$$

The generalized effective stress $\bar{\sigma}_{e}$ is given by:

$$
\bar{\sigma}_{e}=\sqrt{\sigma_{e}^{2}+\frac{1}{1-h_{2} \zeta}\left(h_{2} \eta^{2} \sigma_{p}^{2}+\zeta \sigma_{q}^{2}+2 \eta \sigma_{p} \sigma_{q}\right)}
$$

with:

$$
\sigma_{e}=\sqrt{\frac{3}{2} \boldsymbol{\sigma}: \boldsymbol{M}: \boldsymbol{\sigma}} ; \quad \sigma_{p}=\frac{1}{3} \boldsymbol{\sigma}: \boldsymbol{X} ; \quad \sigma_{q}=\boldsymbol{\sigma}: \boldsymbol{Q}
$$

where $\boldsymbol{Q}$ is a deviatoric second-order tensor, $\boldsymbol{X}$ is a second-order tensor that depends on the shape of the voids through a parameter $\alpha_{2}$, see Eq. (24), and $\boldsymbol{M}$ is the anisotropy tensor proposed by Hill (1948). 


$$
\begin{aligned}
& \boldsymbol{Q}=\left[\begin{array}{ccc}
\frac{1}{2} & 0 & 0 \\
0 & \frac{1}{2} & 0 \\
0 & 0 & -1
\end{array}\right] \\
& \boldsymbol{X}=\left[\begin{array}{ccc}
\frac{3}{2}\left(1-\alpha_{2}\right) & 0 & 0 \\
0 & \frac{3}{2}\left(1-\alpha_{2}\right) & 0 \\
0 & 0 & 3 \alpha_{2}
\end{array}\right] \\
& \boldsymbol{M}=\left[\begin{array}{cccccc}
G+H & -H & -G & 0 & 0 & 0 \\
-H & F+H & -F & 0 & 0 & 0 \\
-G & -F & F+G & 0 & 0 & 0 \\
0 & 0 & 0 & 2 L & 0 & 0 \\
0 & 0 & 0 & 0 & 2 M & 0 \\
0 & 0 & 0 & 0 & 0 & 2 N
\end{array}\right]
\end{aligned}
$$

$\kappa=\sqrt{\frac{6 f(1-f)}{(1+g)(f+g)\left[\left(\beta_{1}-f \beta_{2}\right)\left(h_{1}+3 h_{2}-4 h_{3}\right)+6\left[\alpha_{1}\left(1-\alpha_{1}\right)-f \alpha_{2}\left(1-\alpha_{2}\right)\right] h_{2}+\left[1-\alpha_{1}-f\left(1-\alpha_{2}\right)\right]\left(h_{1}-3 h_{2}+4 h_{3}\right)\right]}}$

131

$$
\begin{gathered}
\zeta=\frac{\kappa^{2}(1+g)(f+g)\left(\alpha_{2}-\alpha_{1}\right)^{2}}{(1-f)^{2}} \\
\eta=\frac{\kappa^{2}(1+g)(f+g)\left(\alpha_{2}-\alpha_{1}\right)}{1-f}
\end{gathered}
$$

132 and the void shape parameters $g, \alpha_{i}$ and $\beta_{i}$, with $i=1,2$, see Eqs. (12), (18) and (20)-(22), are:

$$
g= \begin{cases}0 & \text { (prolate) } \\ \frac{h_{1}+3 h_{2}+4 h_{3}}{2 h_{1}+h_{2}+2 h_{3}} \frac{15 \pi}{8\left(1+\alpha_{1}^{2}\right)} \frac{e_{2}^{3}}{\sqrt{1-e_{2}^{2}}} & \text { (oblate) }\end{cases}
$$




$$
\begin{gathered}
\alpha_{i}= \begin{cases}\frac{1-e_{i}^{2}}{e_{i}^{3}} \operatorname{arctanh}\left(e_{i}\right)-\frac{1-e_{i}^{2}}{e_{i}^{2}} & \text { (prolate) } \\
-\frac{\sqrt{1-e_{i}^{2}}}{e_{i}^{3}} \arctan \left(\frac{e_{i}}{\sqrt{1-e_{i}^{2}}}\right)+\frac{1}{e_{i}^{2}} & \text { (oblate) }\end{cases} \\
\beta_{i}= \begin{cases}\left(1-3 \alpha_{i}\right) \frac{1}{e_{i}^{2}} & \text { (prolate) } \\
-\left(1-3 \alpha_{i}\right) \frac{1-e_{i}^{2}}{e_{i}^{2}} & \text { (oblate) }\end{cases}
\end{gathered}
$$

Notice that $\alpha_{i}$ as defined in equation (24) relates with $\alpha_{i}^{\text {Gologanu }}$ introduced by Gologanu et al. $(1993,1994)$ as $\alpha_{i}^{\text {Gologanu }}=\frac{1-\alpha_{i}}{2}$.

Moreover, the parameters $h_{i}$, with $i=1,2,3$, see Eqs. (15), (20) and (23), are given by:

$$
h_{1}=\frac{1}{4}\left(2 H_{11}+2 H_{22}-H_{33}+2 H_{66}\right) ; \quad h_{2}=\frac{3}{2} H_{33} ; \quad h_{3}=\frac{1}{2}\left(H_{44}+H_{55}\right)
$$

where the constants $H_{11}, H_{22}, H_{33}, H_{44}, H_{55}$, and $H_{66}$ are components of the tensor $\boldsymbol{H}$ which fulfills the condition $\boldsymbol{H}: \boldsymbol{M}=\boldsymbol{K}$, where $\boldsymbol{K}=\boldsymbol{I}-\frac{1}{3}(\mathbf{1} \otimes \mathbf{1})$, with $\boldsymbol{I}$ being the unit fourth-order tensor, so that the following expressions are obtained:

$$
\begin{gathered}
H_{11}=\frac{1}{9} \frac{4 F+G+H}{F G+F H+G H} ; \quad H_{22}=\frac{1}{9} \frac{F+4 G+H}{F G+F H+G H} ; \quad H_{33}=\frac{1}{9} \frac{F+G+4 H}{F G+F H+G H} \\
H_{44}=\frac{1}{L} ; \quad H_{55}=\frac{1}{M} ; \quad H_{66}=\frac{1}{N}
\end{gathered}
$$

Assuming the incompressibility of the matrix material, the evolution of the void volume fraction is given as:

$$
\dot{f}=(1-f) \boldsymbol{d}^{p}: \mathbf{1}
$$

Moreover, following Gologanu et al. (1993, 1994) and Keralavarma and Benzerga (2010), the void shape parameter $S$ evolves according to:

$$
\dot{S}=\frac{3}{2} d_{33}^{p^{\prime}}+\frac{1}{2}\left(\frac{3 \alpha_{1}-1}{f}+1-3 \alpha_{2}\right) \boldsymbol{d}^{p}: \mathbf{1}
$$

${ }_{144}$ where $d_{33}^{p^{\prime}}$ is the deviatoric part of the plastic strain rate along the symmetry axis of the voids $x_{3}$, see Fig. 1 , with $\boldsymbol{d}^{p^{\prime}}=\boldsymbol{d}^{p}-\frac{1}{3}\left(\boldsymbol{d}^{p}: \mathbf{1}\right) \mathbf{1}$ 


\begin{tabular}{cll}
\hline Symbol & Property and units & Value \\
\hline$\rho_{0}$ & Initial density $\left(\mathrm{kg} / \mathrm{m}^{3}\right)$, Eq. (58) & 7600 \\
$E$ & Young's modulus (GPa), Eq. (8) & 70 \\
$\nu$ & Poisson's ratio, Eq. (8) & 0.3 \\
$\sigma_{y 0}$ & Reference yield stress, Eq. (13) & 300 \\
$n$ & Strain hardening sensitivity, Eq. (13) & 0.1 \\
$\varepsilon_{0}$ & Reference strain, Eq. (13) & 0.00429 \\
$f_{0}$ & Initial void volume fraction & $0.001,0.01,0.1$ \\
$K$ & Anisotropy coefficient & $0.75,1,1.5$ \\
$S_{0}$ & Initial void shape parameter & 1.098 (prolate), 0 (spherical) and -1.098 (oblate) \\
\hline
\end{tabular}

Table 1: Material parameters. Data after Srivastava et al. (2014) and dos Santos et al. (2019b).

\subsection{Specialization of the constitutive equations: transversely isotropic matrix material}

The calculations presented in Section 4 consider the matrix material to be transversely isotropic, so that the anisotropy coefficients of the tensor $\boldsymbol{M}$, see Eq. (19), fulfill the following relations (e.g., see Section 2.1. of dos Santos et al. (2019b) for details):

$$
F=G ; \quad N=G+2 H ; \quad L=M=1
$$

leading to:

$$
F=G=\frac{1}{3 K^{2}} ; \quad H=\frac{2 K^{2}-1}{3 K^{2}} ; \quad N=\frac{4 K^{2}-1}{3 K^{2}}
$$

where $K=\sqrt{\frac{G+H}{2 G}}$ is the sole independent anisotropy coefficient. According to Hill (1948), $K \geq \sqrt{\frac{1}{2}}$ (see also Section 2.1 in dos Santos et al. (2019b)), being the isotropic material for $K=1$.

The assumption of a transversely isotropic material represents many practical applications (Hill, 1948), and it is particularly convenient from a modeling standpoint because it allows to derive an axisymmetric cylindrical cavitation model when the material is isotropic in the $r-\theta$ plane, see Section 3. For the specific formulation developed in Section 3, if $K>1$ the yield stress of the material is greater parallel to the axis of the cylindrical cavity, and if $K<1$ the yield stress is greater in the $r-\theta$ plane.

\subsection{Material parameters}

Table 1 shows the values of the initial density and the elastic constants, and the values of the parameters corresponding to the flow strength of the matrix material, which have been taken from Srivastava et al. (2014) and dos Santos et al. (2019b). In Section 4, we carry out a parametric analysis to investigate the effect of plastic anisotropy, initial void volume fraction and initial void shape in the cavitation fields. In the parametric analysis different values of $K, f_{0}$ and $S_{0}$ are used, see Table 1 . The values of $K, f_{0}$ are the same considered by dos Santos et al. (2019b), and the values for the initial aspect ratio of the voids are taken from Monchiet et al. (2008). 


\section{Dynamic cylindrical cavity expansion model}

The problem is stated as that of a circular cylindrical cavity subjected to internal pressure expanding dynamically under plane strain conditions in an infinite porous medium described with the constitutive model presented in Section 2. The novelty lies in the extension of the earlier model of dos Santos et al. (2019b) to consider oblate and prolate voids. As stated in Section 2.3, the matrix material is considered to be transversely isotropic, so that the symmetry axis of both material and voids is parallel to the longitudinal direction of the cylindrical cavity, and therefore the principal directions of stress and strain coincide. Moreover, the artificial viscosity approach derived by Lew et al. (2001) is used to capture the plastic shock waves that emerge at high cavity expansion velocities, preventing the formation of discontinuities in the elastoplastic fields that develop near the cavity, and facilitating the numerical integration of the set of differential equations which govern the problem (dos Santos et al., 2019a,b).

The cavity expansion model is formulated relative to an Eulerian cylindrical coordinate system $(r, \theta, z)$ with origin located at the center of the cavity, see Fig. 2, using notations customarily considered (e.g. see Masri and Durban (2009) and Cohen and Durban (2013), among others). Following the works of Durban and Fleck (1997) and Durban and Masri (2004), the cavity, of instantaneous radius $a$, is assumed to expand at constant velocity under self-similar, steady-state conditions, so that the only independent variable of the problem is the dimensionless radial coordinate $\xi=r / a$. Accordingly, the derivative with respect to the radial coordinate becomes:

$$
\frac{d()}{d r}=\frac{d()}{d \xi} \frac{d \xi}{d r}=()^{\prime} \frac{1}{a}
$$

where the prime superscript denotes differentiation with respect to $\xi$. In addition, the time derivative can be transformed by the following self-similarity relation (Durban and Fleck, 1997; Masri and Durban, 2005):

$$
\dot{()}=\frac{d()}{d \xi} \dot{\xi}=()^{\prime} \frac{\dot{a}}{a}(v-\xi)
$$

where $v=\dot{r} / \dot{a}$ is the dimensionless radial velocity, with $\dot{a}=$ constant (constant velocity expansion), so that the dimensionless acceleration reads $\dot{v}=\ddot{r} / \dot{a}$.

The principal values of the rate of deformation tensor are:

$$
\begin{gathered}
d_{r r}=\frac{d \dot{r}}{d r}=\frac{\dot{a}}{a} v^{\prime} \\
d_{\theta \theta}=\frac{\dot{r}}{r}=\frac{\dot{a}}{a} \frac{v}{\xi}
\end{gathered}
$$




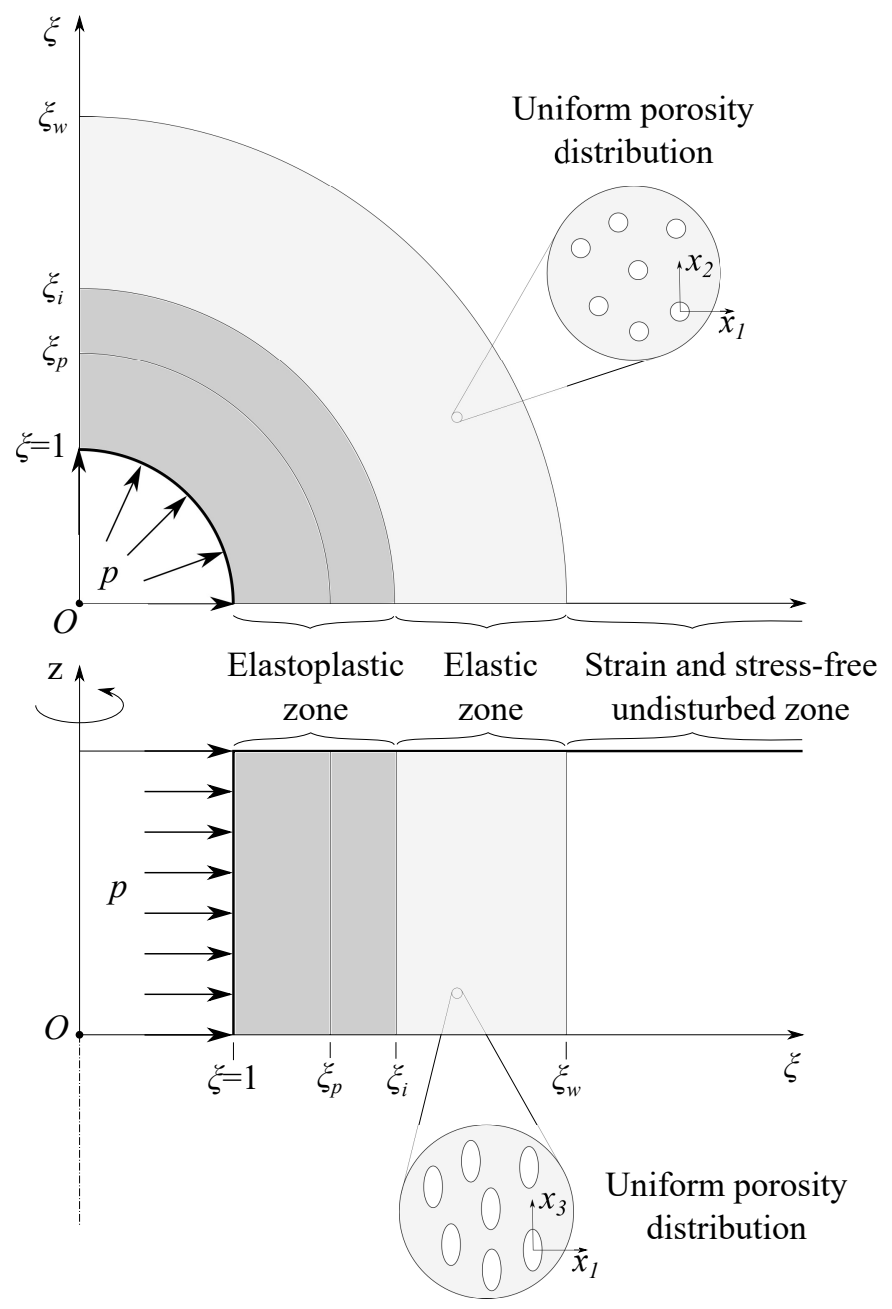

Figure 2: Schematic of self-similar field developed during dynamic expansion of a circular cylindrical cavity in an elastoplastic porous medium with spheroidal voids. The internal pressure $p$ is applied at the cavity wall where $\xi=1$. The remote field, at $\xi>\xi_{w}$, is undisturbed while behind the elastic wave front, located at $\xi=\xi_{w}$, an elastic range develops. The transition between elastic and elastoplastic regions is denoted by $\xi_{i}$, and the location of the plastic shock wave that emerges at high cavity expansion velocities by $\xi_{p}$. 


$$
d_{z z}=0
$$

$$
\begin{aligned}
& N_{r r}=\frac{1}{2} \frac{\frac{3\left(G\left(\Sigma_{r r}-\Sigma_{z z}\right)+H\left(\Sigma_{r r}-\Sigma_{\theta \theta}\right)\right)}{\Sigma_{y}}+\phi \frac{\Sigma_{p}}{\Sigma_{y}}+\chi \frac{\Sigma_{q}}{\Sigma_{y}}+\kappa\left(1-\alpha_{2}\right)(g+1)(f+g) \sinh \left(\frac{\kappa \Sigma_{p}}{\Sigma_{y}}\right)}{\left(\frac{\Sigma_{e}}{\Sigma_{y}}\right)^{2}+(g+1)(f+g) \sinh \left(\frac{\kappa \Sigma_{p}}{\Sigma_{y}}\right) \frac{\kappa \Sigma_{p}}{\Sigma_{y}}} \\
& N_{\theta \theta}=\frac{1}{2} \frac{\frac{3\left(F\left(\Sigma_{\theta \theta}-\Sigma_{z z}\right)+H\left(\Sigma_{\theta \theta}-\Sigma_{r r}\right)\right)}{\Sigma_{y}}+\phi \frac{\Sigma_{p}}{\Sigma_{y}}+\chi \frac{\Sigma_{q}}{\Sigma_{y}}+\kappa\left(1-\alpha_{2}\right)(g+1)(f+g) \sinh \left(\frac{\kappa \Sigma_{p}}{\Sigma_{y}}\right)}{\left(\frac{\bar{\Sigma}_{e}}{\Sigma_{y}}\right)^{2}+(g+1)(f+g) \sinh \left(\frac{\kappa \Sigma_{p}}{\Sigma_{y}}\right) \frac{\kappa \Sigma_{p}}{\Sigma_{y}}} \\
& N_{z z}=\frac{1}{2} \frac{\frac{3\left(F\left(\Sigma_{z z}-\Sigma_{\theta \theta}\right)+G\left(\Sigma_{z z}-\Sigma_{r r}\right)\right)}{\Sigma_{y}}+2 \varphi \frac{\Sigma_{p}}{\Sigma_{y}}+2 \psi \frac{\Sigma_{q}}{\Sigma_{y}}+2 \alpha \alpha_{2} \kappa(g+1)(f+g) \sinh \left(\frac{\kappa \Sigma_{p}}{\Sigma_{y}}\right)}{\left(\frac{\bar{\Sigma}_{e}}{\Sigma_{y}}\right)^{2}+(g+1)(f+g) \sinh \left(\frac{\kappa \Sigma_{p}}{\Sigma_{y}}\right) \frac{\kappa \Sigma_{p}}{\Sigma_{y}}}
\end{aligned}
$$




$$
\phi=\frac{\left(1-\alpha_{2}\right) h_{2} \eta^{2}+\eta}{1-h_{2} \zeta} ; \quad \chi=\frac{\left(1-\alpha_{2}\right) \eta+\zeta}{1-h_{2} \zeta} ; \quad \varphi=\frac{\alpha_{2} h_{2} \eta^{2}-\eta}{1-h_{2} \zeta} ; \quad \psi=\frac{\alpha_{2} \eta-\zeta}{1-h_{2} \zeta}
$$

where $\Sigma_{r r}=\sigma_{r r} / E, \Sigma_{\theta \theta}=\sigma_{\theta \theta} / E$ and $\Sigma_{z z}=\sigma_{z z} / E$ are the dimensionless radial, circumferential, and axial stresses, respectively, $\bar{\Sigma}_{e}=\bar{\sigma}_{e} / E$ is the dimensionless generalized effective stress and $\Sigma_{y}=\sigma_{y} / E$ is the dimensionless yield strength of the matrix material. Moreover, $\Sigma_{p}=\sigma_{p} / E$ and $\Sigma_{q}=\sigma_{q} / E$ are dimensionless scalar stress measures.

The additive decomposition of the rate of deformation tensor, see equation (6), can be rewritten using expressions (32)-(34), (35)-(37) and (38)-(40), as follows:

$$
\begin{aligned}
& v^{\prime}=(v-\xi)\left(\Sigma_{r r}^{\prime}-\nu\left(\Sigma_{\theta \theta}^{\prime}+\Sigma_{z z}^{\prime}\right)+(1-f) \frac{\partial \bar{\varepsilon}^{p}}{\partial \Sigma_{y}} \Sigma_{y}^{\prime} N_{r r}\right) \\
& \frac{v}{\xi}=(v-\xi)\left(\Sigma_{\theta \theta}^{\prime}-\nu\left(\Sigma_{z z}^{\prime}+\Sigma_{r r}^{\prime}\right)+(1-f) \frac{\partial \bar{\varepsilon}^{p}}{\partial \Sigma_{y}} \Sigma_{y}^{\prime} N_{\theta \theta}\right) \\
& 0=(v-\xi)\left(\Sigma_{z z}^{\prime}-\nu\left(\Sigma_{r r}^{\prime}+\Sigma_{\theta \theta}^{\prime}\right)+(1-f) \frac{\partial \bar{\varepsilon}^{p}}{\partial \Sigma_{y}} \Sigma_{y}^{\prime} N_{z z}\right)
\end{aligned}
$$

where the relation $3 \Sigma_{h}=\Sigma_{r r}+\Sigma_{\theta \theta}+\Sigma_{z z}$ and the time derivative (31) have been used. The derivative $\partial \bar{\varepsilon}^{p} / \partial \Sigma_{y}$, which is obtained from equation (13), is calculated as:

$$
\frac{\partial \bar{\varepsilon}^{p}}{\partial \Sigma_{y}}=\frac{\varepsilon_{0}}{n \Sigma_{y 0}}\left(\frac{\Sigma_{y}}{\Sigma_{y 0}}\right)^{\frac{1}{n}-1}
$$

with $\Sigma_{y 0}=\sigma_{y 0} / E$ being the dimensionless initial yield strength of the matrix material.

Moreover, using equations (38)-(40), the expression for the evolution of the void volume fraction given in (26) can be written as follows:

$$
f^{\prime}=(1-f)^{2}\left(\bar{\varepsilon}^{p}\right)^{\prime}\left(N_{r r}+N_{\theta \theta}+N_{z z}\right)
$$

where the time derivative (31) has been used.

Similarly, using equations (31) and (38)-(40), the evolution of the void shape parameter given in (27) can be expressed as:

$$
S^{\prime}=(1-f)\left(\bar{\varepsilon}^{p}\right)^{\prime}\left(\left(N_{z z}-N_{r r}\right)+\frac{1}{2}\left(\frac{3 \alpha_{1}-1}{f}+1-3 \alpha_{2}\right)\left(N_{r r}+N_{\theta \theta}+N_{z z}\right)\right)
$$

The balance of mass takes the form: 


$$
\dot{\rho}+\rho \operatorname{tr}(\boldsymbol{d})=0
$$

which can be rewritten as:

$$
(v-\xi) \frac{\rho^{\prime}}{\rho}+v^{\prime}+\frac{v}{\xi}=0
$$

using equations (31)-(34). Moreover, inserting expressions (45) and (46) into equation (52) leads to:

$$
\frac{\rho^{\prime}}{\rho}=-\left(3(1-2 \nu) \Sigma_{h}^{\prime}+\frac{f^{\prime}}{(1-f)}\right)
$$

where expression (49) has been used. Following Cohen and Durban (2013), equation (53) is integrated from a given coordinate $\xi$ to the elastic wave front $\xi_{w}$, so that the following algebraic relation for the density ratio is obtained:

$$
\frac{\rho}{\rho_{0}}=\left(\frac{1-f}{1-f_{0}}\right) \exp \left(-3(1-2 \nu) \Sigma_{h}\right)
$$

Moreover, the balance of linear momentum along the radial direction takes the form:

$$
\frac{\partial \sigma_{r r}^{t}}{\partial r}+\frac{\sigma_{r r}^{t}-\sigma_{\theta \theta}^{t}}{r}=\rho \ddot{r}
$$

where $\sigma_{r r}^{t}=\sigma_{r r}+\sigma_{r r}^{v}$ and $\sigma_{\theta \theta}^{t}=\sigma_{\theta \theta}+\sigma_{\theta \theta}^{v}$, with $\sigma_{r r}^{v}$ and $\sigma_{\theta \theta}^{v}$ being the artificial viscous radial and circumferential stresses defined as (Wilkins, 1980; Lew et al., 2001):

$$
\begin{array}{r}
\sigma_{r r}^{v}= \begin{cases}c_{0}^{2} \rho(\Delta r)^{2}\left|d_{r r}\right| d_{r r}+c_{L} \rho \Delta r V_{s} d_{r r} & \text { for } d_{r r}<0 \\
0 & \text { for } d_{r r} \geq 0\end{cases} \\
\sigma_{\theta \theta}^{v}= \begin{cases}c_{0}^{2} \rho(\Delta r)^{2}\left|d_{\theta \theta}\right| d_{\theta \theta}+c_{L} \rho \Delta r V_{s} d_{\theta \theta} & \text { for } d_{\theta \theta}<0 \\
0 & \text { for } d_{\theta \theta} \geq 0\end{cases}
\end{array}
$$

where $c_{0} \approx 2$ and $c_{L} \approx 1$ are constant parameters, $\Delta r$ is a given increment along the radial direction and $V_{s}=\sqrt{E / \rho}$ (see dos Santos et al. (2019a) for details).

Using expressions (30) and (31), equation (55) can be rewritten as:

$$
\left(\Sigma_{r r}^{t}\right)^{\prime}+\frac{1}{\xi}\left(\Sigma_{r r}^{t}-\Sigma_{\theta \theta}^{t}\right)=m^{2} \frac{\rho}{\rho_{0}}(v-\xi) v^{\prime}
$$


where $\Sigma_{r r}^{t}=\Sigma_{r r}+\Sigma_{r r}^{v}$ and $\Sigma_{\theta \theta}^{t}=\Sigma_{\theta \theta}+\Sigma_{\theta \theta}^{v}$, with $\Sigma_{r r}^{v}=\sigma_{r r}^{v} / E$ and $\Sigma_{\theta \theta}^{v}=\sigma_{\theta \theta}^{v} / E$. Notice that the artificial viscosity approach is only used in the definition of the stresses entering into the balance of linear momentum (see dos Santos et al. (2019a,b) for details). Moreover, $m=\frac{\dot{a}}{\sqrt{E / \rho_{0}}}$ is the dimensionless cavitation velocity. Section 4 shows results for $0.05 \leq m \leq 0.4$, which correspond to dimensional cavitation velocities ranging between $0.1574 \mathrm{Km} / \mathrm{s}$ and $1.2139 \mathrm{Km} / \mathrm{s}$ for the material parameters given in Table 1. Similar range of cavity expansion velocities was investigated by Cohen and Durban (2013) and dos Santos et al. (2019a).

The problem is completed by the following boundary conditions:

$$
\begin{gathered}
v=1 \quad \text { and } \quad \Sigma_{r r}=-P \text { at } \xi=1 \\
v=0 \quad \text { and } \quad \Sigma_{r r}=\Sigma_{\theta \theta}=0 \text { at } \xi=\xi_{w}
\end{gathered}
$$

where $P=\frac{p}{E}$ is the dimensionless applied pressure. An iterative shooting method is employed to enforce the velocity boundary condition, while the stress boundary condition is used to calculate the corresponding applied pressure. The initial conditions, with an unknown constant, and the location of the elastic wave front are determined adapting the elastic solution derived by Masri and Durban (2009) to the constitutive framework used in this paper (see also dos Santos et al. (2019b)).

\subsection{Plastic shock condition}

The condition for the plastic shock formation is derived adapting the approach of Cohen and Durban (2013) to the constitutive model presented in Section 2.

Firstly, the flow potential, Eq. (12), is linearized as follows:

$$
\begin{aligned}
\Phi^{\prime} & \approx \frac{\partial \Phi}{\partial \Sigma_{r r}} \Sigma_{r r}^{\prime}+\frac{\partial \Phi}{\partial \Sigma_{\theta \theta}} \Sigma_{\theta \theta}^{\prime}+\frac{\partial \Phi}{\partial \Sigma_{z z}} \Sigma_{z z}^{\prime}+\left(\frac{\partial \Phi}{\partial \Sigma_{y}}+\frac{\partial \Phi}{\partial f}(1-f)^{2}\left(N_{r r}+N_{\theta \theta}+N_{z z}\right) \frac{\partial \bar{\varepsilon}^{p}}{\partial \Sigma_{y}}\right) \Sigma_{y}^{\prime} \\
& +\frac{\partial \Phi}{\partial S}(1-f)\left(N_{z z}-\frac{1}{2}\left(N_{r r}+N_{\theta \theta}\right)+\frac{1}{2}\left(\frac{3 \alpha_{1}-1}{f}+1-3 \alpha_{2}\right)\left(N_{r r}+N_{\theta \theta}+N_{z z}\right)\right) \frac{\partial \bar{\varepsilon}^{p}}{\partial \Sigma_{y}} \Sigma_{y}^{\prime} \approx 0
\end{aligned}
$$

where equations (49) and (50) have been used, and the shape functions $\eta, \zeta, \kappa$ and $g$ have been considered to be constant within a small variation of plastic deformation. The derivative $\frac{\partial \Phi}{\partial S}$ is calculated numerically, while the expressions for $\frac{\partial \Phi}{\partial \Sigma_{r r}}, \frac{\partial \Phi}{\partial \Sigma_{\theta \theta}}, \frac{\partial \Phi}{\partial \Sigma_{z z}}, \frac{\partial \Phi}{\partial \Sigma_{y}}$ and $\frac{\partial \Phi}{\partial f}$ are given by:

$$
\frac{\partial \Phi}{\partial \Sigma_{r r}}=\frac{3\left(G\left(\Sigma_{r r}-\Sigma_{z z}\right)+H\left(\Sigma_{r r}-\Sigma_{\theta \theta}\right)\right)}{\Sigma_{y}^{2}}+\frac{\left(\phi \Sigma_{p}+\chi \Sigma_{q}\right)}{\Sigma_{y}^{2}}+\frac{\kappa}{\Sigma_{y}}\left(1-\alpha_{2}\right)(1+g)(f+g) \sinh \left(\frac{\kappa \Sigma_{p}}{\Sigma_{y}}\right)
$$




$$
\begin{gathered}
\frac{\partial \Phi}{\partial \Sigma_{\theta \theta}}=\frac{3\left(H\left(\Sigma_{\theta \theta}-\Sigma_{r r}\right)+F\left(\Sigma_{\theta \theta}-\Sigma_{z z}\right)\right)}{\Sigma_{y}^{2}}+\frac{\left(\phi \Sigma_{p}+\chi \Sigma_{q}\right)}{\Sigma_{y}^{2}}+\frac{\kappa}{\Sigma_{y}}\left(1-\alpha_{2}\right)(1+g)(f+g) \sinh \left(\frac{\kappa \Sigma_{p}}{\Sigma_{y}}\right) \\
\frac{\partial \Phi}{\partial \Sigma_{z z}}=\frac{3\left(F\left(\Sigma_{z z}-\Sigma_{\theta \theta}\right)+G\left(\Sigma_{z z}-\Sigma_{r r}\right)\right)}{\Sigma_{y}^{2}}+\frac{2\left(\varphi \Sigma_{p}+\psi \Sigma_{q}\right)}{\Sigma_{y}^{2}}+2 \alpha_{2} \frac{\kappa}{\Sigma_{y}}(1+g)(f+g) \sinh \left(\frac{\kappa \Sigma_{p}}{\Sigma_{y}}\right) \\
\frac{\partial \Phi}{\partial \Sigma_{y}}=-2\left((1+g)(f+g) \sinh \left(\frac{\kappa \Sigma_{p}}{\Sigma_{y}}\right) \frac{\kappa \Sigma_{p}}{\Sigma_{y}^{2}}+\frac{\bar{\Sigma}_{e}^{2}}{\Sigma_{y}^{3}}\right) \\
\frac{\partial \Phi}{\partial f}=2\left((1+g) \cosh \left(\frac{\kappa \Sigma_{p}}{\Sigma_{y}}\right)-(f+g)\right)
\end{gathered}
$$

Secondly, the determinant of the matrix of coefficients of the derivatives with respect to $\xi$ in Eqs. (45)-(47), (58), and (61) is imposed to be zero, leading to the following singularity condition:

$$
\Delta=m^{2} \frac{\rho}{\rho_{0}}(v-\xi)^{2}-\frac{\frac{(1-f)}{(1+\nu)} \frac{\partial \bar{\varepsilon}^{p}}{\partial \Sigma_{y}}\left(\left(N_{\theta \theta}+\nu N_{z z}\right) \frac{\partial \Phi}{\partial \Sigma_{\theta \theta}}+\left(N_{z z}+\nu N_{\theta \theta}\right) \frac{\partial \Phi}{\partial \Sigma_{z z}}\right)-(1-\nu)\left(\frac{\partial \Phi}{\partial \Sigma_{y}}+\omega_{f} \frac{\partial \Phi}{\partial f}+\omega_{S} \frac{\partial \Phi}{\partial S}\right)}{(1-f) \frac{\partial \bar{\varepsilon}^{p}}{\partial \Sigma_{y}}\left(\omega_{r r} \frac{\partial \Phi}{\partial \Sigma_{r r}}+\omega_{\theta \theta} \frac{\partial \Phi}{\partial \Sigma_{\theta \theta}}+\omega_{z z} \frac{\partial \Phi}{\partial \Sigma_{z z}}\right)-(1-2 \nu)(1+\nu)\left(\frac{\partial \Phi}{\partial \Sigma_{y}}+\omega_{f} \frac{\partial \Phi}{\partial f}+\omega_{S} \frac{\partial \Phi}{\partial S}\right)}=0
$$

with:

$$
\begin{gathered}
\omega_{r r}=(1-\nu) N_{r r}+\nu\left(N_{\theta \theta}+N_{z z}\right) \\
\omega_{\theta \theta}=(1-\nu) N_{\theta \theta}+\nu\left(N_{r r}+N_{z z}\right) \\
\omega_{z z}=(1-\nu) N_{z z}+\nu\left(N_{r r}+N_{\theta \theta}\right) \\
\omega_{f}=(1-f)^{2} \frac{\partial \bar{\varepsilon}^{p}}{\partial \Sigma_{y}}\left(N_{r r}+N_{\theta \theta}+N_{z z}\right) \\
\omega_{S}=(1-f)\left(N_{z z}-\frac{1}{2}\left(N_{r r}+N_{\theta \theta}\right)+\frac{1}{2}\left(\frac{3 \alpha_{1}-1}{f}+1-3 \alpha_{2}\right)\left(N_{r r}+N_{\theta \theta}+N_{z z}\right)\right) \frac{\partial \bar{\varepsilon}^{p}}{\partial \Sigma_{y}}
\end{gathered}
$$

Hereinafter, $\Delta$ will be called the shock wave function, and the dimensionless cavity expansion velocity for

which the plastic shock wave emerges will be referred to as critical dimensionless cavitation velocity $m_{c}$. The 
location of the plastic shock wave corresponds to the radial coordinate for which the condition $\Delta=0$ is met within the elastoplastic zone $1 \leq \xi \leq \xi_{i}$, see Fig. 2 .

\section{Results}

This section of the paper is split into two parts. The effect of initial void shape in the elastoplastic fields that develop near the cavity is investigated in Section 4.1, and the effect of anisotropy in Section 4.2.

\subsection{The effect of initial void shape}

Fig. 3 displays the variation in dimensionless applied pressure $P$ with dimensionless cavitation velocity $m$ for three different values of the initial void shape parameter: $S_{0}=1.098$ (prolate with $\frac{a_{1}}{b_{1}}=3$ ), $S_{0}=0$ (spherical with $\frac{a_{1}}{b_{1}}=1$ ) and $S_{0}=-1.098$ (oblate with $\frac{a_{1}}{b_{1}}=0.33$ ), see equation (4) and Fig. 1. All the results presented in this section correspond to initial void volume fraction $f_{0}=0.01$ and anisotropy parameter $K=1$ (isotropic plasticity). The $P-m$ curves feature a concave-upward shape, consistent with the cylindrical cavity expansion results presented by Masri and Durban (2009) and dos Santos et al. (2019b) for fully dense materials and for porous materials with spherical voids, respectively. The novelty brought to light in this paper is that the effect of the initial shape of the voids in the $P-m$ curves is negligible.

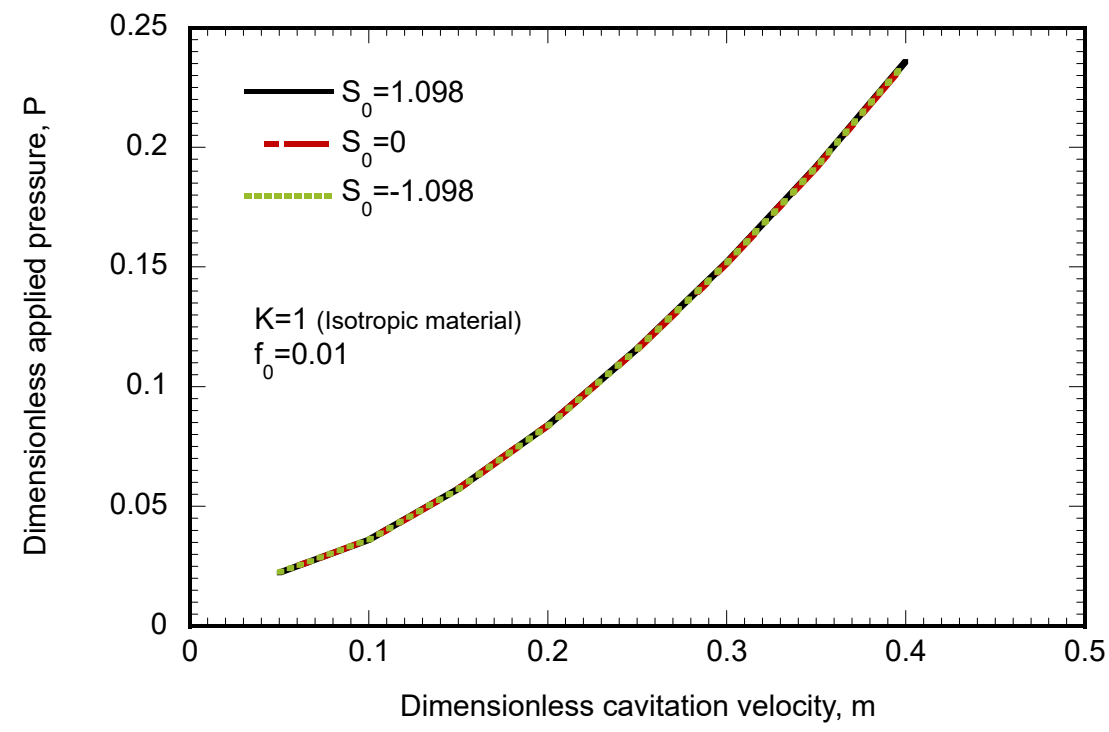

Figure 3: Variation in dimensionless applied pressure $P$ with dimensionless cavitation velocity $m$ for three different values of the initial void shape parameter: $S_{0}=1.098$ (prolate with $\frac{a_{1}}{b_{1}}=3$ ), $S_{0}=0$ (spherical with $\frac{a_{1}}{b_{1}}=1$ ) and $S_{0}=-1.098$ (oblate with $\frac{a_{1}}{b_{1}}=0.33$ ), see equation (4) and Fig. 1. The initial void volume fraction is $f_{0}=0.01$, and the anisotropy parameter is $K=1$ (isotropic plasticity).

In contrast, the initial shape of the voids affects the elastoplastic fields that develop near the cavity. Fig. 4 illustrates the variation in (a) dimensionless radial stress $\Sigma_{r r}$, (b) dimensionless circumferential stress $\Sigma_{\theta \theta}$, (c) dimensionless axial stress $\Sigma_{z z}$, (d) dimensionless generalized effective stress $\bar{\Sigma}_{e}$ and (e) void volume fraction $f$ with the dimensionless radial coordinate $\xi$. The dimensionless cavitation velocity is $m=0.2$. As in Fig. 3 , calculations 
have been performed for $S_{0}=1.098, S_{0}=0$ and $S_{0}=-1.098$. Notice that, while the cavitation fields obtained for prolate and initially spherical voids are very similar, significant differences appear for oblate voids at intermediate values of the dimensionless radial coordinate.

Fig. 4(a) shows that the dimensionless radial stress decreases monotonically with the radial coordinate. At $\xi=1$, the absolute value of the radial stress matches with the cavity pressure. The elastic/elastoplastic interface is located at $\xi_{i} \approx 4.4$. The location of the elastic/elastoplastic interface is taken as the smaller radial coordinate for which the plastic strains are zero. The calculations performed with initially spherical and prolate voids show a sudden drop in the radial stress for an intermediate value of the radial coordinate which indicates the emergence of a plastic shock wave at $\xi_{p} \approx 3.75$. Recall from Section 3.1 that the location of the shock wave corresponds to the radial coordinate for which the condition $\Delta=0$ is met within the elastoplastic zone $1 \leq \xi \leq \xi_{i}$. Note that the change in the radial stress induced by the shock, while abrupt, is not a discontinuity. The artificial viscosity included in the model provides the shock wave with a finite width (dos Santos et al., 2019b). On the other hand, the calculations performed with oblate voids predict a more gentle decrease of the radial stress, with no indications of the formation of plastic shocks (the shock wave function does not meet the condition $\Delta=0$ within the elastoplastic zone).

Figs. 4(b) and 4(c) show the circumferential and axial stress fields. The $\Sigma_{\theta \theta}-\xi$ and $\Sigma_{z z}-\xi$ curves are qualitatively and quantitatively similar to the radial stress fields (note that $\Sigma_{h} \approx-0.08$ at the cavity wall). The calculations performed with initially spherical and prolate voids display a sudden drop in the circumferential and axial stress which reveals the formation of a shock wave, while the rate of decrease of $\Sigma_{\theta \theta}$ and $\Sigma_{z z}$ within the elastoplastic range is largely constant for the calculations performed with oblate voids.

Fig. 4(d) displays the evolution of the dimensionless generalized effective stress with the radial coordinate. Near the cavity wall, $\bar{\Sigma}_{e}$ is one order of magnitude less than the radial, circumferential and axial stresses -compare Figs. 4(a), 4(b), 4(c) and 4(d)-, i.e., the hydrostatic stress is one order of magnitude greater than the effective stress. The shock wave formation in the calculations for $S_{0}=1.098$ and $S_{0}=0$ leads to a sudden drop of effective stress, which partially bounces back immediately after, leading to a short plateau that extends up to the elastic/elastoplastic interface. In the calculation performed with $S_{0}=-1.098$, the drop of dimensionless generalized effective stress that occurs at $\xi \approx 2.9$ does not indicate a shock wave - the condition $\Delta=0$ is not met and the artificial viscosity is not required to integrate the set of differential equations which govern the cavity expansion model-, but the total collapse of the oblate voids (see the discussion below).

Fig. 4(e) illustrates the evolution of the void volume fraction with the radial coordinate. For the initially prolate and spherical voids, the void volume fraction is zero between the cavity wall and the plastic shock location $1 \leq \xi \leq \xi_{p}$. Within the shock, the value of $f$ suddenly increases, reaching the initial void volume fraction $f_{0}=0.01$ at the elastic/elastoplastic interface. The model predicts that for $S_{0}=1.098$ and $S_{0}=0$ the voids become increasingly prolate during the compaction process and eventually collapse with $S$ growing unbounded 
(the evolution of $S$ with $\xi$ is not shown for the sake of brevity). The calculation corresponding to $S_{0}=-1.098$ reveals that the total collapse of the initially oblate voids occurs at $\xi \approx 2.9$, i.e., at shorter distance from the cavity wall. In addition, the closure of the initially oblate voids is not abrupt but gradual and continuous until the elastic/elastoplastic interface is reached. The model predicts that for $S_{0}=-1.098$ the voids become increasingly oblate during the closure process and eventually collapse with $S$ decreasing unbounded.

The influence of the initial voids shape in the elastoplastic fields that develop near the cavity is significantly reduced for lower and higher cavitation velocities. For instance, Fig. 5 pictures the dimensionless generalized effective stress $\bar{\Sigma}_{e}$ and the void volume fraction $f$ versus the dimensionless radial coordinate $\xi$ for smaller cavitation velocity $m=0.05$. The $\bar{\Sigma}_{e}-\xi$ curves obtained for prolate, spherical and oblate voids virtually overlap each other, see Fig. 5(a). Moreover, while the closure of the voids starts slightly nearer the cavity for oblate voids, the differences between the $f-\xi$ curves obtained for the three values of $S_{0}$ considered are small, see Fig. 5(b).

Fig. 6 shows the evolution of $\bar{\Sigma}_{e}$ and $f$ versus the dimensionless radial coordinate $\xi$ for greater cavity expansion velocity $m=0.4$. The results for the dimensionless generalized effective stress pictured in Fig. 6(a) show that a shock wave is formed at $\xi_{p} \approx 2.25$ for the three initial void shapes considered, including for oblate voids $S_{0}=-1.098$, for which no plastic shock was observed at $m=0.2$. This is an original result of this investigation which indicates that the initial shape of the voids affects the cavity expansion velocity for which plastic shocks first emerge. We will further elaborate on this issue in the following paragraphs. The $f-\xi$ curves reported in Fig. 6(b) show that the void volume fraction increases rapidly from zero to the initial void volume fraction within the shock wave for prolate, spherical and oblate voids. The differences in the porosity evolution for the three values of $S_{0}$ considered are smaller than for lower cavity expansion velocities, see Figs. 4(e) and 5(b), which suggests that the influence of the initial shape of the voids in the porosity field becomes less when plastic shocks emerge.

Fig. 7 shows the variation in the location of the elastic/elastoplastic interface $\xi_{i}$ and the shock wave $\xi_{p}$ with the dimensionless cavitation velocity $m$. Consistent with the results presented by dos Santos et al. (2019b), both elastic/elastoplastic interface and plastic shock are located closer to the cavity wall as the cavitation velocity increases. The evolution of $\xi_{i}$ and $\xi_{p}$ with $m$ displays a concave-upward shape, so that as the cavity expansion velocity increases, the location of the plastic shock is nearer to the elastic/elastoplastic interface (this result is consistent with the theoretical predictions of Cohen et al. (2010)). Note that the effect of the initial voids shape $S_{0}$ on the location of the elastic/elastoplastic interface is very small. However, as anticipated in the previous paragraph, the minimum cavity expansion velocity at which the shocks form, i.e. the critical cavitation velocity $m_{c}$, depends on the initial shape of the voids. Namely, Fig. 7 shows that $m_{c}$ is $0.255,0.160$ and 0.150 , for $S_{0}=-1.098, S_{0}=0$ and $S_{0}=1.098$, respectively. The values of $m_{c}$ are calculated with the condition $\Delta=0$, see equation (67), and coincide with the minimum cavity expansion velocity for which the artificial viscosity approach, 


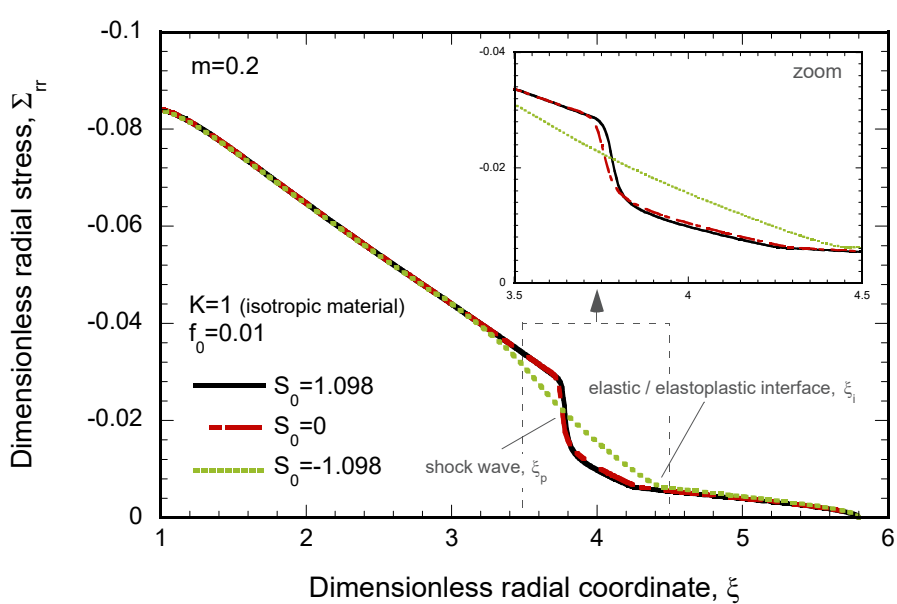

(a)

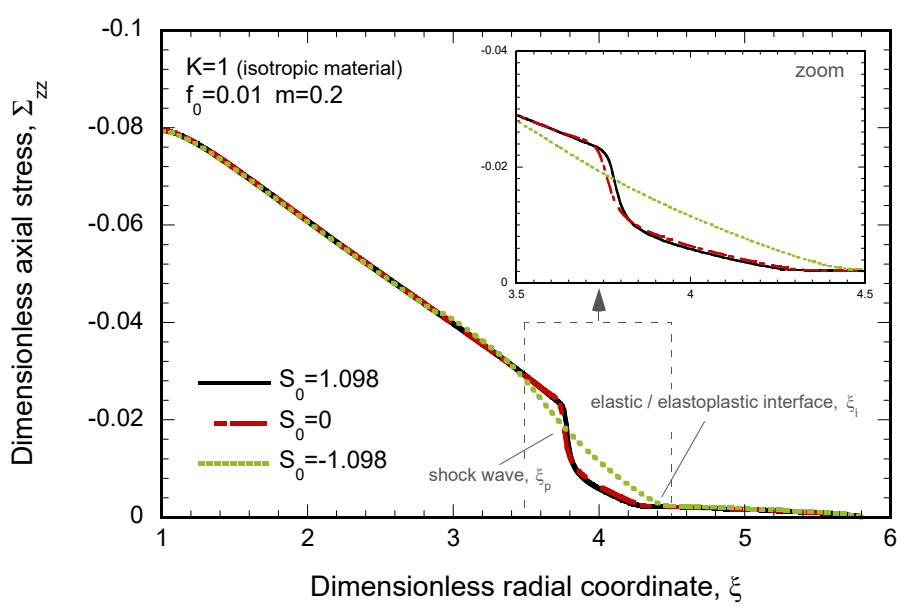

(c)

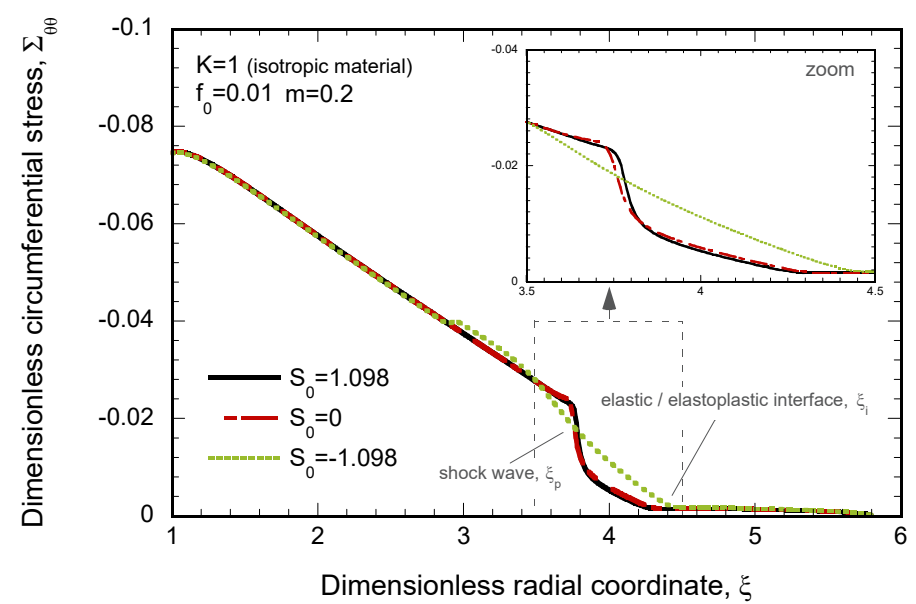

(b)

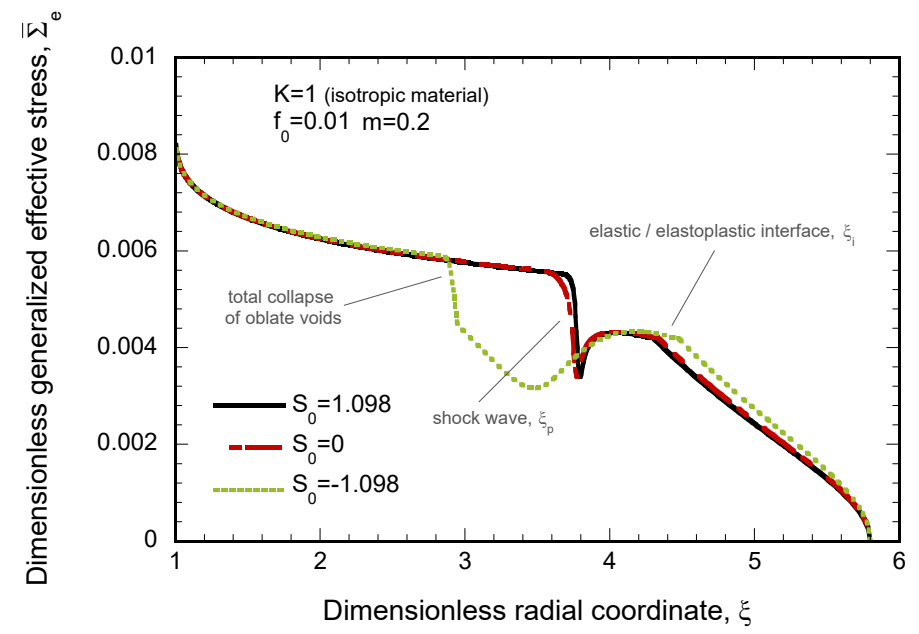

(d)

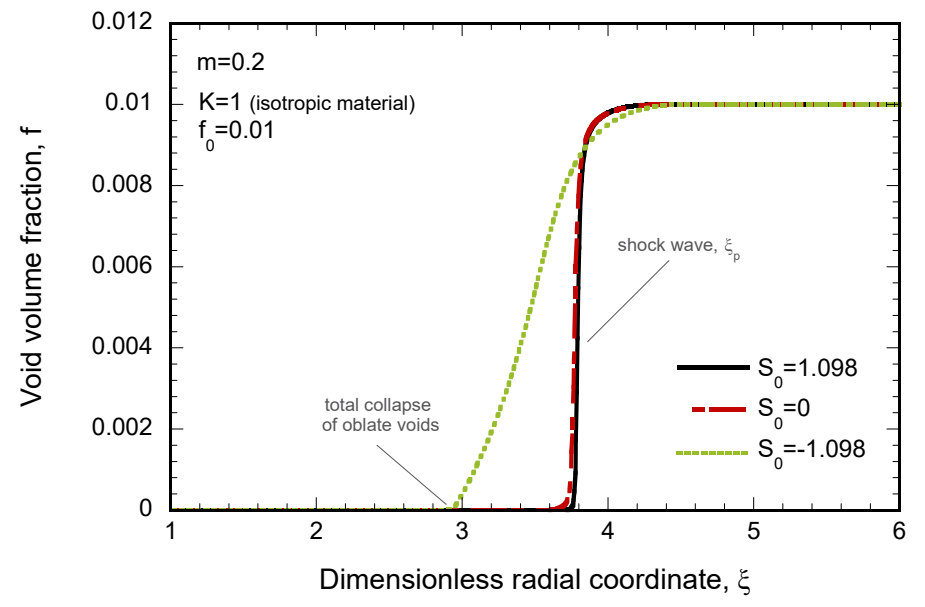

(e)

Figure 4: Results corresponding to three different values of the initial void shape parameter: $S_{0}=1.098$ (prolate with $\frac{a_{1}}{b_{1}}=3$ ), $S_{0}=0$ (spherical with $\frac{a_{1}}{b_{1}}=1$ ) and $S_{0}=-1.098$ (oblate with $\frac{a_{1}}{b_{1}}=0.33$ ), see equation (4) and Fig. 1 . The initial void volume fraction is $f_{0}=0.01$, and the anisotropy parameter is $K=1$ (isotropic plasticity). The dimensionless cavitation velocity is $m=0.2$. Variation in (a) dimensionless radial stress $\Sigma_{r r}$, (b) dimensionless circumferential stress $\Sigma_{\theta \theta}$, (c) dimensionless axial stress $\Sigma_{z z}$, (d) dimensionless generalized effective stress $\bar{\Sigma}_{e}$ and (e) void volume fraction $f$ with dimensionless radial coordinate $\xi$. 


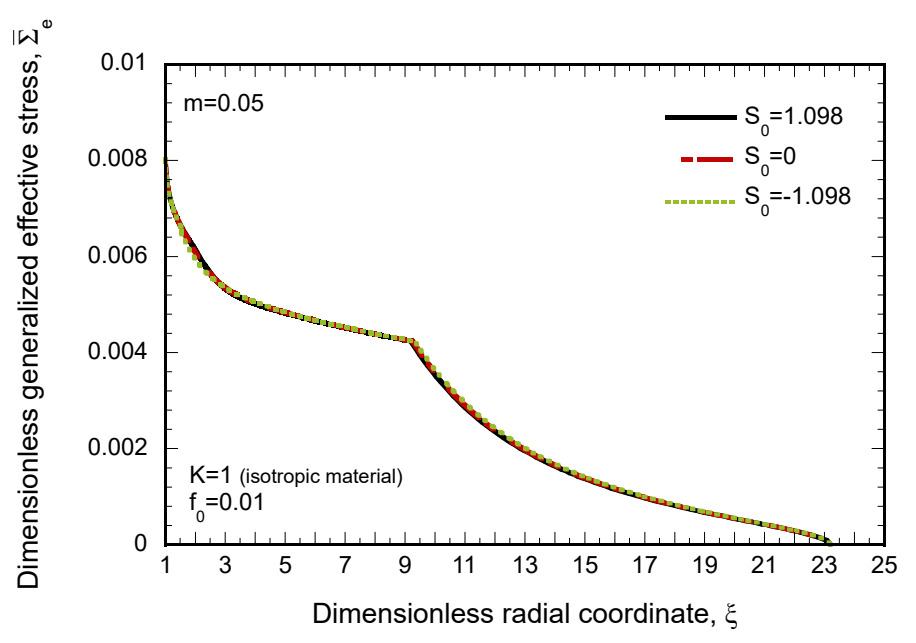

(a)

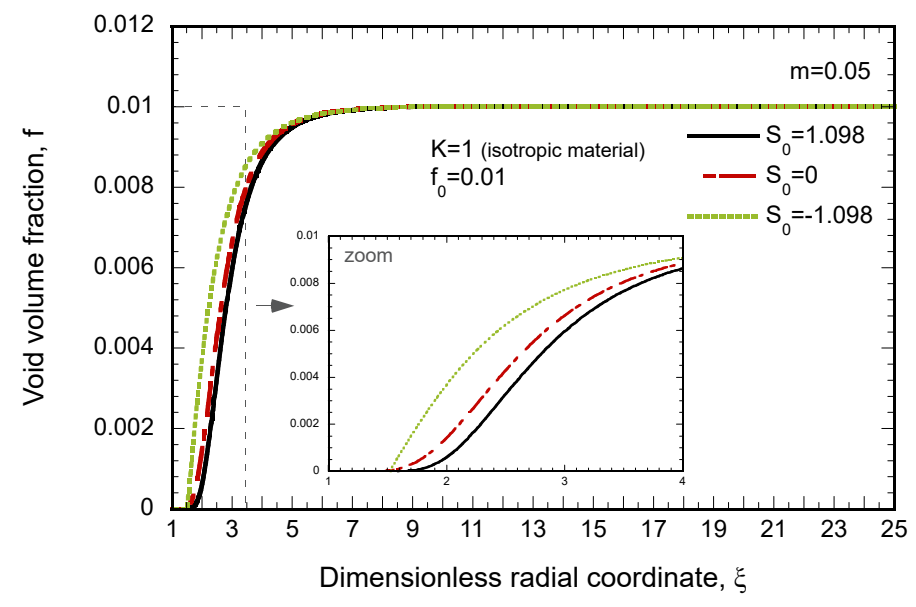

(b)

Figure 5: Results corresponding to three different values of the initial void shape parameter: $S_{0}=1.098$ (prolate with $\frac{a_{1}}{b_{1}}=3$ ), $S_{0}=0$ (spherical with $\frac{a_{1}}{b_{1}}=1$ ) and $S_{0}=-1.098$ (oblate with $\frac{a_{1}}{b_{1}}=0.33$ ), see equation (4) and Fig. 1 . The initial void volume fraction is $f_{0}=0.01$, and the anisotropy parameter is $K=1$ (isotropic plasticity). The dimensionless cavitation velocity is $m=0.05$. Variation in (a) dimensionless generalized effective stress $\bar{\Sigma}_{e}$ and (b) void volume fraction $f$ with dimensionless radial coordinate $\xi$.

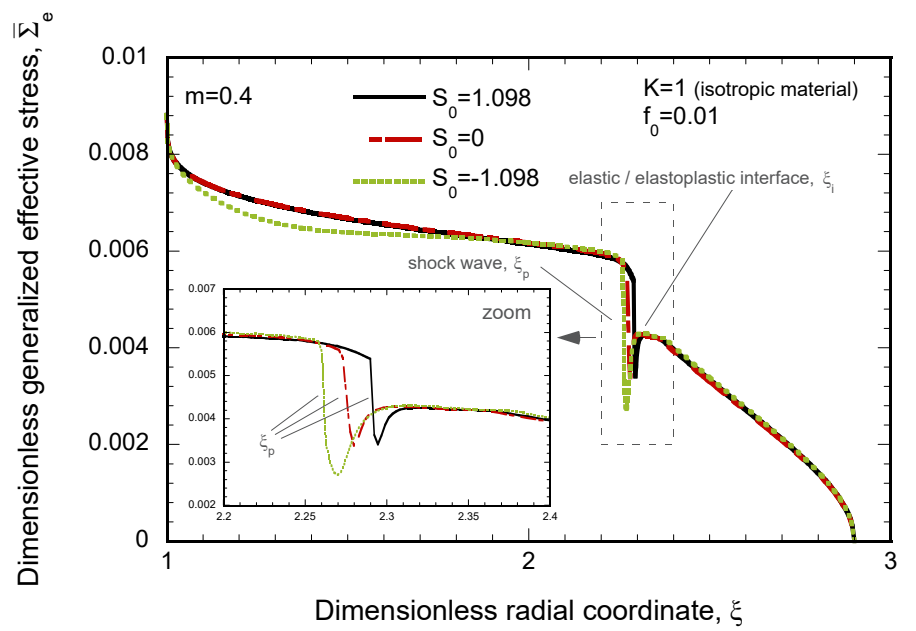

(a)

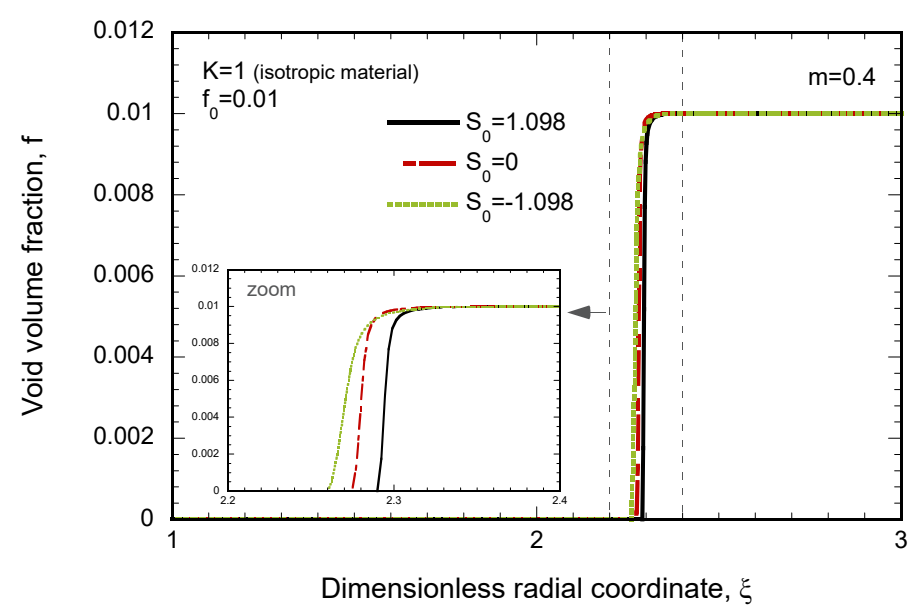

(b)

Figure 6: Results corresponding to three different values of the initial void shape parameter: $S_{0}=1.098$ (prolate with $\frac{a_{1}}{b_{1}}=3$ ), $S_{0}=0$ (spherical with $\frac{a_{1}}{b_{1}}=1$ ) and $S_{0}=-1.098$ (oblate with $\frac{a_{1}}{b_{1}}=0.33$ ), see equation (4) and Fig. 1 . The initial void volume fraction is $f_{0}=0.01$, and the anisotropy parameter is $K=1$ (isotropic plasticity). The dimensionless cavitation velocity is $m=0.4$. Variation in (a) dimensionless generalized effective stress $\bar{\Sigma}_{e}$ and (b) void volume fraction $f$ with dimensionless radial coordinate $\xi$. 


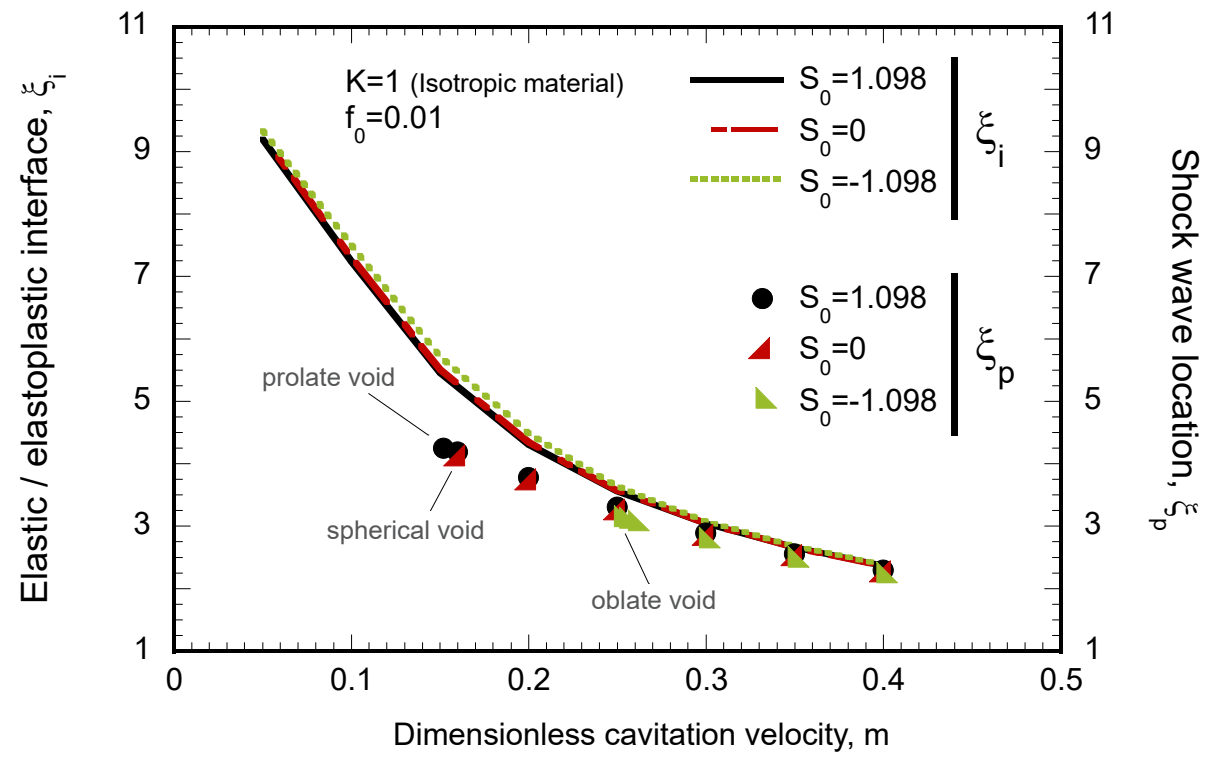

Figure 7: Results corresponding to three different values of the initial void shape parameter: $S_{0}=1.098$ (prolate with $\frac{a_{1}}{b_{1}}=3$ ), $S_{0}=0$ (spherical with $\frac{a_{1}}{b_{1}}=1$ ) and $S_{0}=-1.098$ (oblate with $\frac{a_{1}}{b_{1}}=0.33$ ), see equation (4) and Fig. 1. Variation in the location of the elastic/elastoplastic interface $\xi_{i}$ and the plastic shock wave $\xi_{p}$ with the dimensionless cavitation velocity $m$. The initial void volume fraction is $f_{0}=0.01$, and the anisotropy parameter is $K=1$ (isotropic plasticity).

see equations (56)-(57), is required to solve the set of differential equations which govern the cavity expansion model.

The evolution of $m_{c}$ with $S_{0}$ is further investigated in Fig. 8 for values of the void shape parameter within the range $-1.60 \leq S_{0} \leq 1.60$ (which corresponds to $0.2 \leq \frac{a_{1}}{b_{1}} \leq 5$ ). The cavity expansion model predicts that for initially oblate voids the relationship between $m_{c}$ and $S_{0}$ is nonlinear, featuring a concave-downward shape with a maximum value of $m_{c}$ for $S_{0} \approx-1$. For initially prolate voids, the value of $m_{c}$ displays a mild and roughly linear decrease with the increase of the void shape parameter $S_{0}$. Note that the case of initially spherical voids $S_{0}=0$ corresponds to a kink in the $m_{c}-S_{0}$ curve. These results reinforce the idea that the emergence of shock waves in porous materials is modulated by the shape of the voids. Nevertheless, these predictions are specific for the constitutive model employed in this work, see Section 2, and further research is required to obtain definite conclusions on the effect of voids shape on shocks formation, see the discussion and perspectives for future work reported in Section 5.

\subsection{The effect of anisotropy}

Fig. 9 pictures the variation in dimensionless generalized effective stress $\bar{\Sigma}_{e}$ and void volume fraction $f$ with dimensionless radial coordinate $\xi$ for calculations performed with initially prolate voids $S_{0}=1.098$. The initial void volume fraction is $f_{0}=0.01$ and the dimensionless cavitation velocity is $m=0.2$. A comparison is performed between calculations corresponding to two different values of the anisotropy parameter: $K=0.75$ and $K=1.5$. Recall that if $K>1$ the yield stress of the material is greater along the axis of the cylindrical cavity, and if $K<1$ 


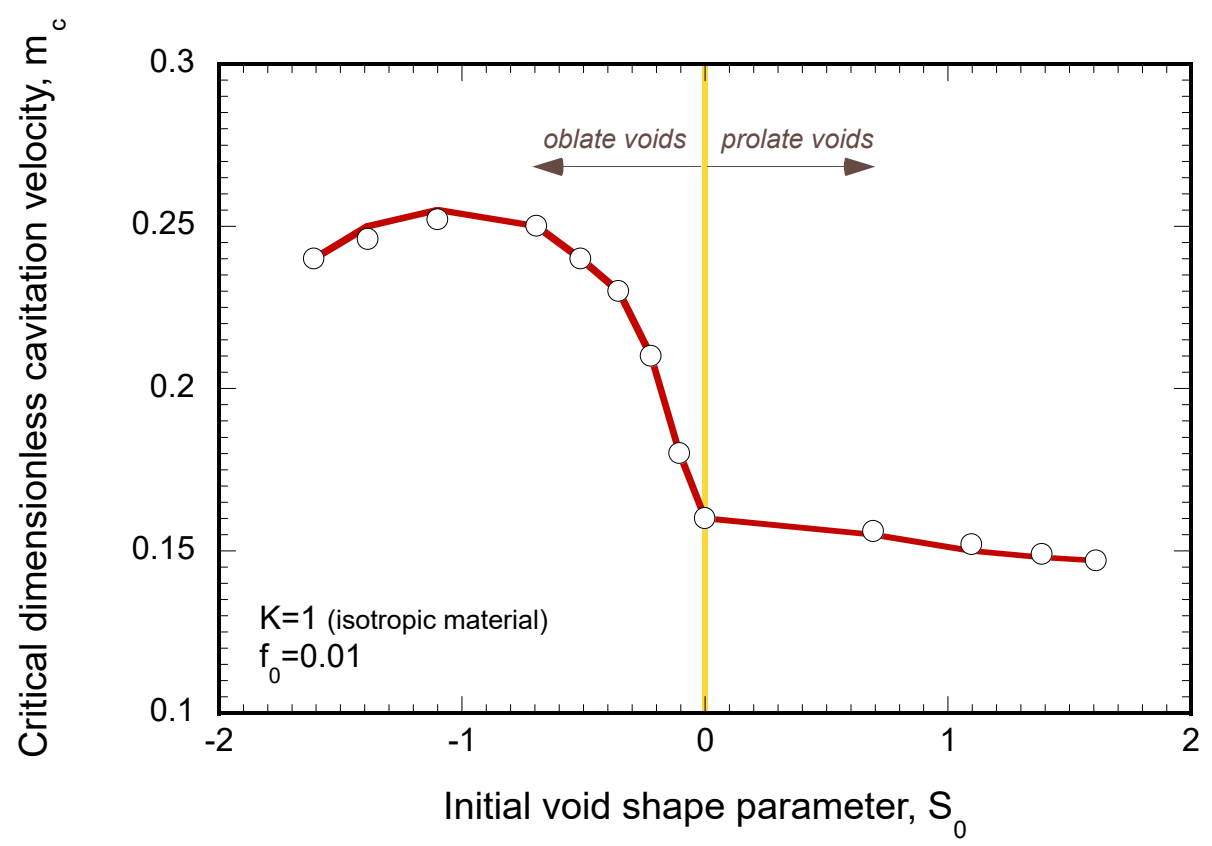

Figure 8: Variation in the critical dimensionless cavitation velocity $m_{c}$ with the initial void shape parameter $S_{0}$, see equation $(4)$ and Fig. 1. The initial void volume fraction is $f_{0}=0.01$, and the anisotropy parameter is $K=1$ (isotropic plasticity).

it is greater in the $r-\theta$ plane. The anisotropy has little effect on the effective stress and porosity fields. The only noticeable difference is that the location of the shock wave and the elastic/elastoplastic interface are slightly further from the cavity wall in the case of $K=1.5$. A shock is formed at $\xi_{p} \approx 3.75$ for both anisotropy parameter values considered.

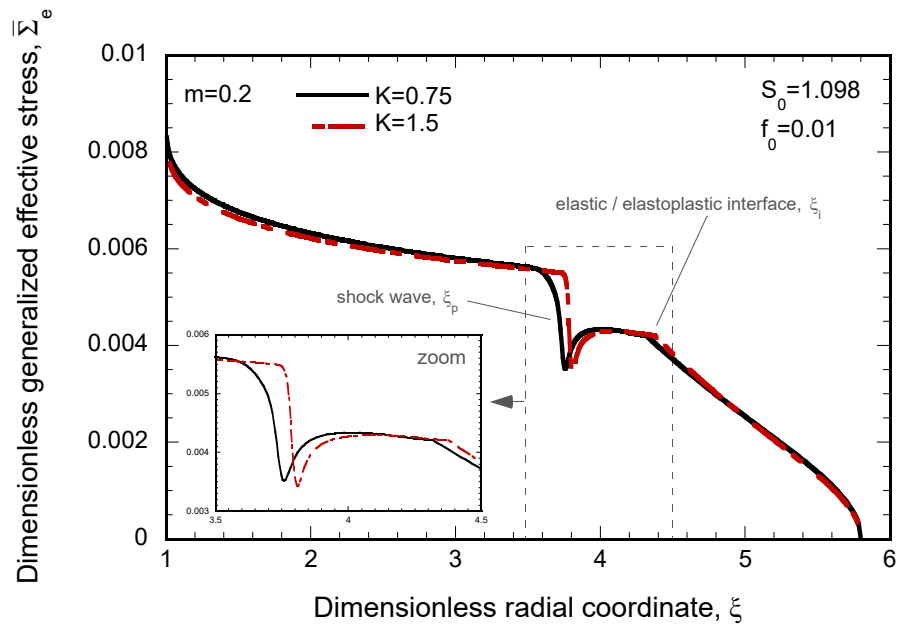

(a)

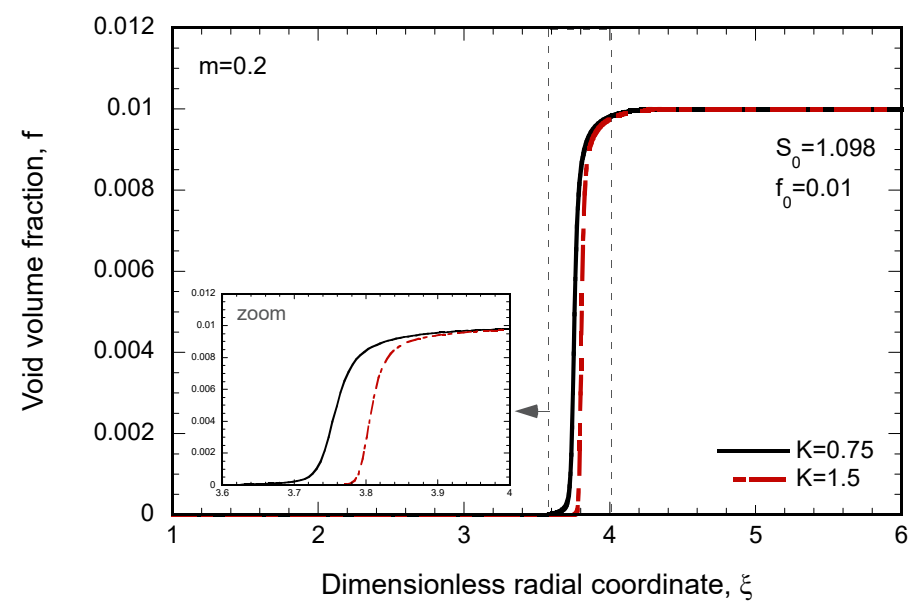

(b)

Figure 9: Results corresponding to prolate voids $S_{0}=1.098\left(\frac{a_{1}}{b_{1}}=3\right)$ for two different values of the anisotropy parameter: $K=0.75$ and $K=1.5$. The initial void volume fraction is $f_{0}=0.01$, and the dimensionless cavitation velocity is $m=0.2$. Variation in (a) dimensionless generalized effective stress $\bar{\Sigma}_{e}$ and (b) void volume fraction $f$ with dimensionless radial coordinate $\xi$.

These results stand in contrast to the calculations performed for initially oblate voids $S_{0}=-1.098$ for the same cavity expansion velocity $m=0.2$, which show a noticeable influence of the anisotropy parameter on the 


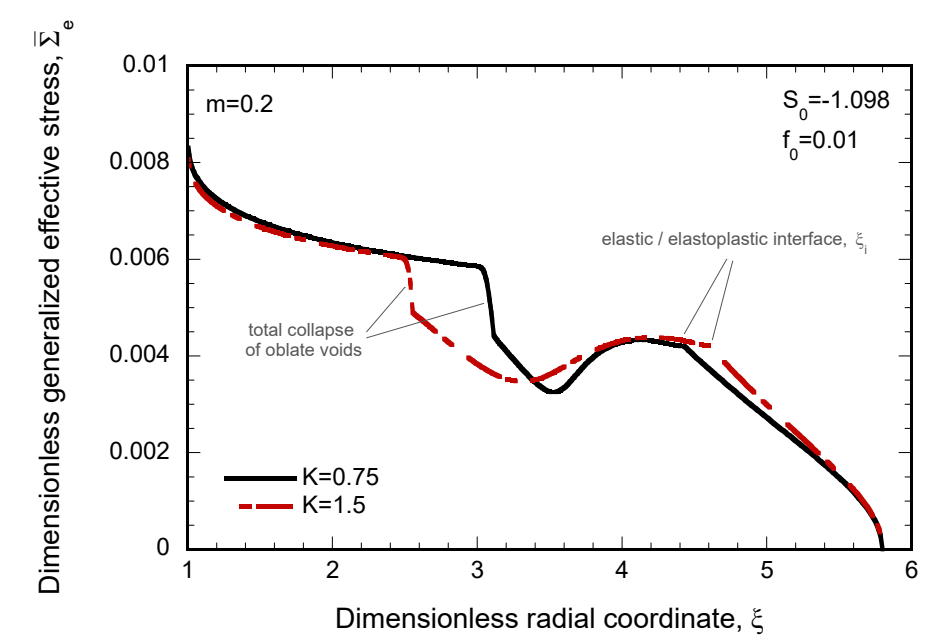

(a) radial coordinate.

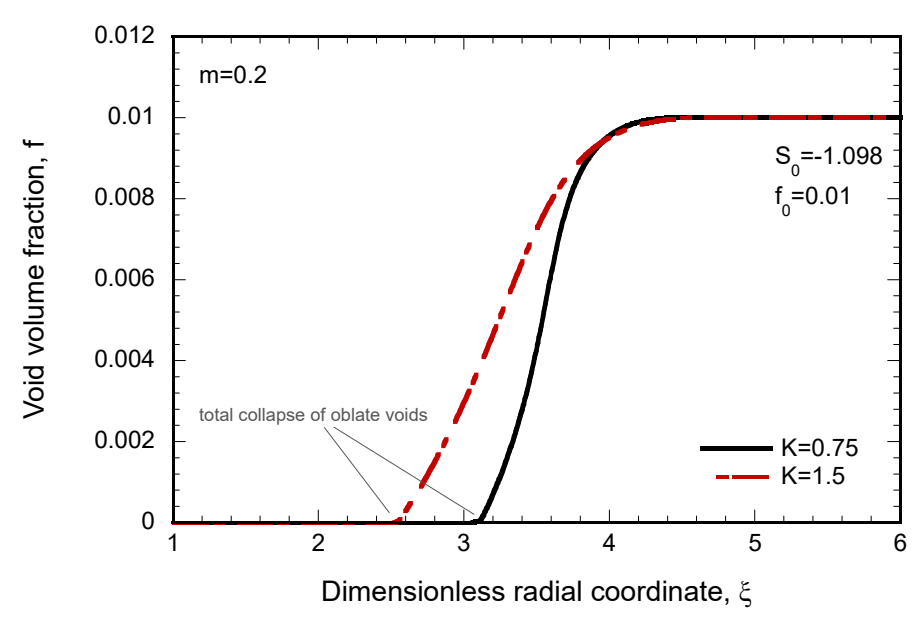

(b)

Figure 10: Results corresponding to oblate voids $S_{0}=-1.098\left(\frac{a_{1}}{b_{1}}=0.33\right)$ for two different values of the anisotropy parameter: $K=0.75$ and $K=1.5$. The initial void volume fraction is $f_{0}=0.01$, and the dimensionless cavitation velocity is $m=0.2$. Variation in (a) dimensionless generalized effective stress $\bar{\Sigma}_{e}$ and (b) void volume fraction $f$ with dimensionless radial coordinate $\xi$.

effective stress and porosity fields, see Fig. 10. Specifically, the drop in the effective stress for intermediate values of the radial coordinate, which corresponds to the total collapse of the voids, occurs further from the cavity for $K=0.75$, see Fig. 10(a). In addition, the closure of the voids with the radial coordinate is more gradual for $K=1.5$, see Fig. 10(b), with the elastic/elastoplastic interface being located at a slightly greater value of the

Nevertheless, the influence of the parameter $K$ in the elastoplastic cavitation fields depends on the cavity expansion velocity, so that the effect of the anisotropy is generally less for other values of $m$.

Results for initially oblate voids $S_{0}=-1.098$ and lower cavity expansion velocity $m=0.05$ are shown in Fig. 11. The $\bar{\Sigma}_{e}-\xi$ curves obtained for both values of $K$ considered are very similar, see Fig. 11(a), the difference only appears in the location of the elastic/elastoplastic interface, which is slightly further from the cavity for $K=1.5$. Moreover, the $f-\xi$ curves pictured in Fig. 11(b) show that the collapse of the voids occurs slightly closer to the cavity for $K=1.5$.

Results for initially oblate voids $S_{0}=-1.098$ and greater cavity expansion velocity $m=0.4$ are shown in Fig. 12. A shock wave is formed at $\xi_{p} \approx 2.25$ for both values of $K$ considered. Small differences in the $\bar{\Sigma}_{e}-\xi$ curves appear within the elastoplastic zone at intermediate values of $\xi$ so that the effective stress for $K=0.75$ is slightly below the predictions obtained for $K=1.5$, see Fig. 12(a). Moreover, the porosity fields for $K=0.75$ and $K=1.5$ are very similar, with a sudden variation in the void volume fraction within the shock, see Fig. 12(b). Consistent with the results presented in Fig. 9(b) for prolate voids, the anisotropy shows little effect on the porosity fields for cavity expansion velocities leading to shock formation.

In contrast, anisotropy does play a significant role on the critical cavitation velocity. Fig. 13 shows the variation 


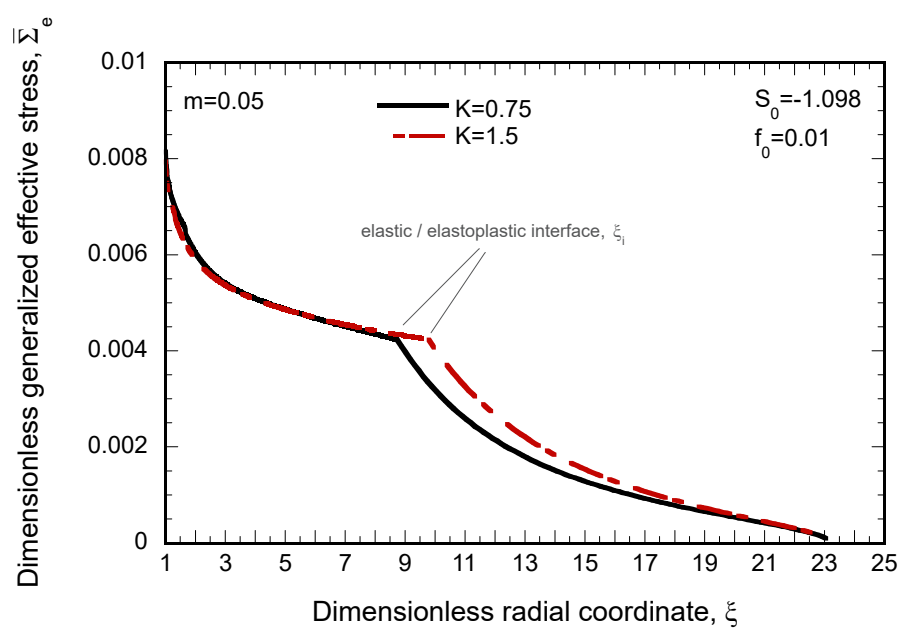

(a)

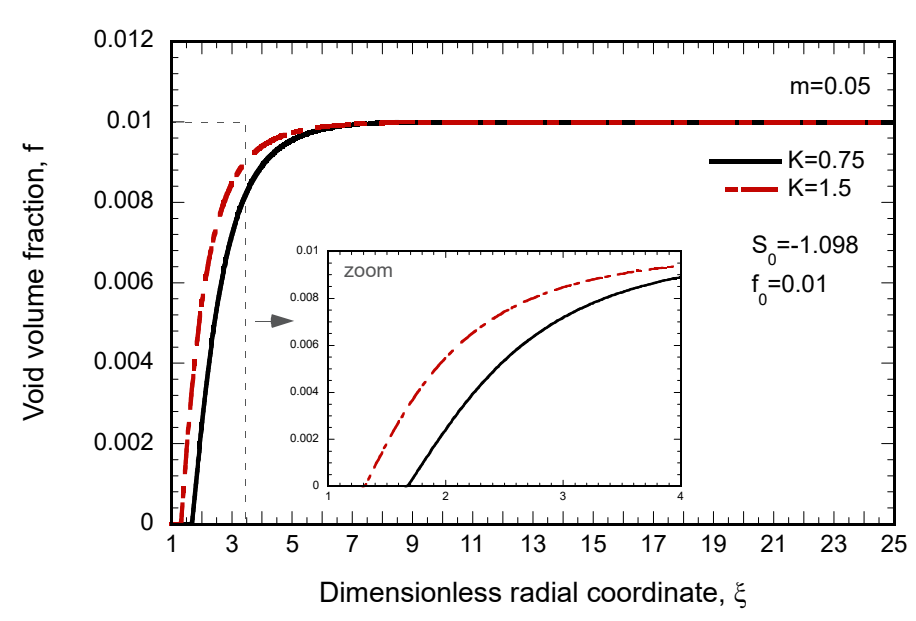

(b)

Figure 11: Results corresponding to oblate voids $S_{0}=-1.098\left(\frac{a_{1}}{b_{1}}=0.33\right)$ for two different values of the anisotropy parameter: $K=0.75$ and $K=1.5$. The initial void volume fraction is $f_{0}=0.01$, and the dimensionless cavitation velocity is $m=0.05$. Variation in (a) dimensionless generalized effective stress $\bar{\Sigma}_{e}$ and (b) void volume fraction $f$ with dimensionless radial coordinate $\xi$.

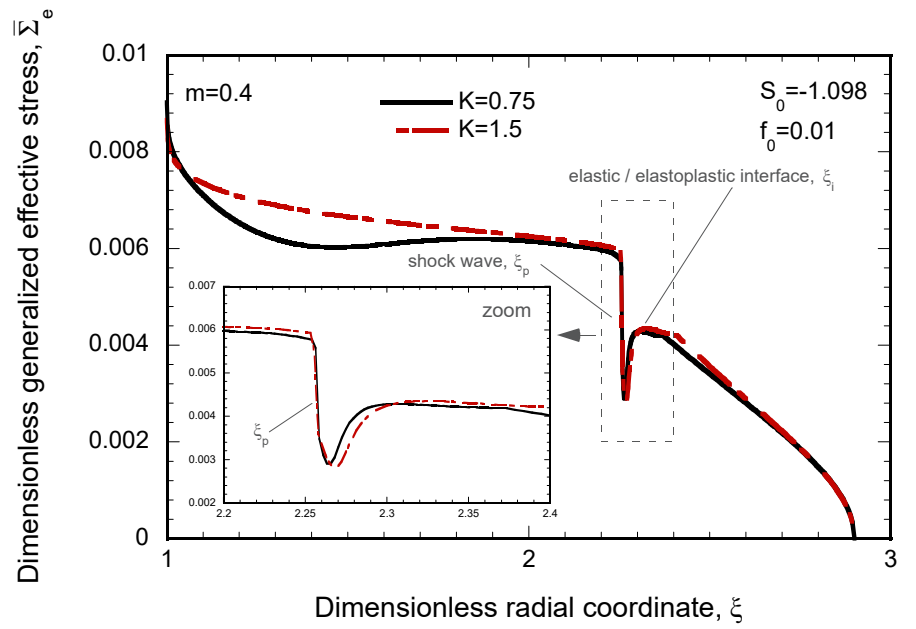

(a)

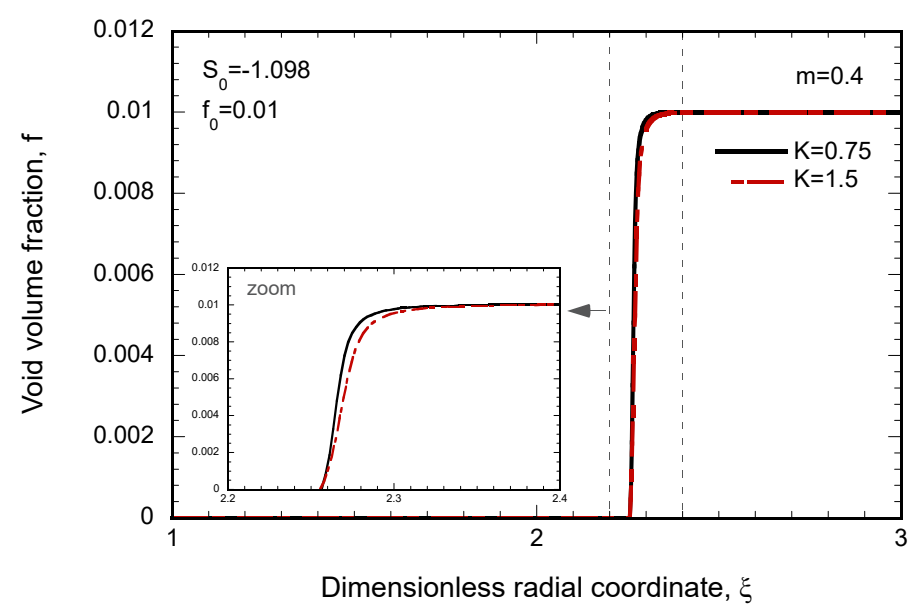

(b)

Figure 12: Results corresponding to oblate voids $S_{0}=-1.098\left(\frac{a_{1}}{b_{1}}=0.33\right)$ for two different values of the anisotropy parameter $K=0.75$ and $K=1.5$. Initial void volume fraction $f_{0}=0.01$ and dimensionless cavitation velocity $m=0.4$. Variation in (a) dimensionless generalized effective stress $\bar{\Sigma}_{e}$ and (b) void volume fraction $f$ with dimensionless radial coordinate $\xi$. 
in $m_{c}$ with the initial void shape parameter $S_{0}$ for $K=0.75$ and $K=1.5$. While the $m_{c}-S_{0}$ curves feature similar shape for both anisotropy parameter values considered, there are quantitative differences in the predictions obtained from the cavity expansion model. Specifically, for initially oblate voids $S_{0}<0$, the formation of shocks occurs for greater value of the cavity expansion velocity for $K=1.5$, and the opposite trend is obtained for initially prolate voids $S_{0}>0$, with the absolute value of the slope of the $S_{0}>0$ branch increasing as $K$ increases. Note that for initially spherical voids $S_{0}=0$, the cavity expansion velocity for which shocks first emerge is roughly the same for both values of $K$. These findings provide new insights into the interplay between voids shape, anisotropy and shock wave formation in porous materials. However, as mentioned before, the predictions of the cavity expansion model are specific for the constitutive framework presented in Section 2, so that further research is needed to broaden the generality of our conclusions.

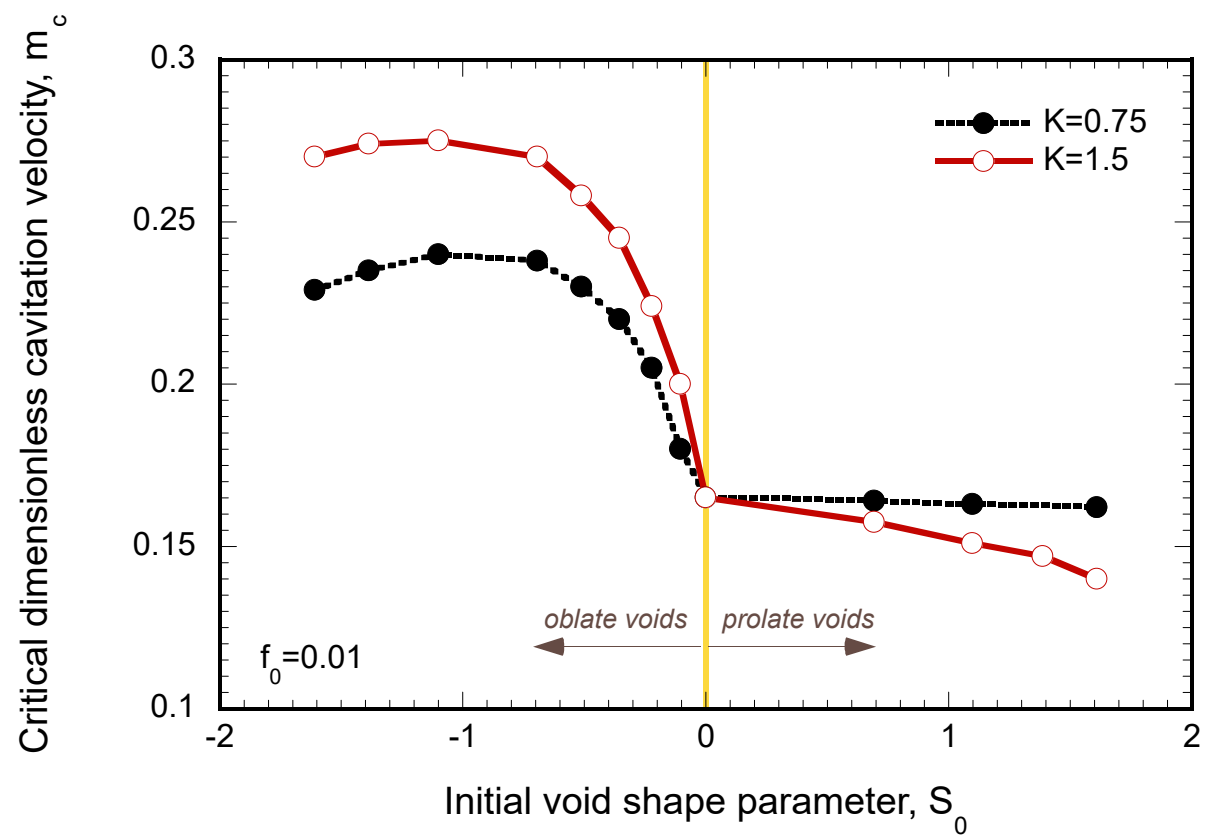

Figure 13: Results corresponding to two different values of the anisotropy parameter: $K=0.75$ and $K=1.5$. Initial void volume fraction $f_{0}=0.01$. Variation in the critical dimensionless cavitation velocity $m_{c}$ with the initial void shape parameter $S_{0}$, see equation (4) and Fig. 1.

The effect of initial porosity on the critical cavitation velocity $m_{c}$ is investigated in Fig. 14 for calculations performed with anisotropy parameter values $K=0.75, K=1$ (isotropic plasticity) and $K=1.5$, and two different initial void volume fractions, $f_{0}=0.001$ and $f_{0}=0.1$ in Figs. 14(a) and 14(b), respectively. There is qualitative agreement between the $m_{c}-S_{0}$ curves obtained for both values of $f_{0}$, and the general trends obtained for the effect of $K$ on the evolution of the critical cavitation velocity with the initial shape of the voids are virtually independent of the initial void volume fraction (see also the results for $f_{0}=0.01$ in Fig. 13). On the other hand, the comparison between the results presented for $f_{0}=0.001$ and $f_{0}=0.1$ shows that decreasing/increasing the initial porosity delays/promotes the formation of shocks waves (Cohen and Durban, 2013), so that the $m_{c}-S_{0}$ 


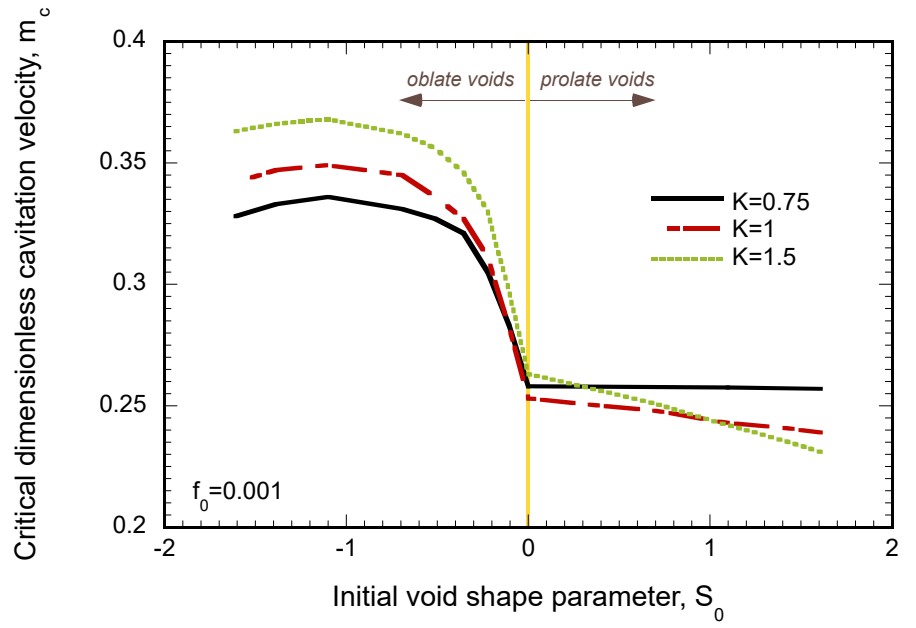

(a)

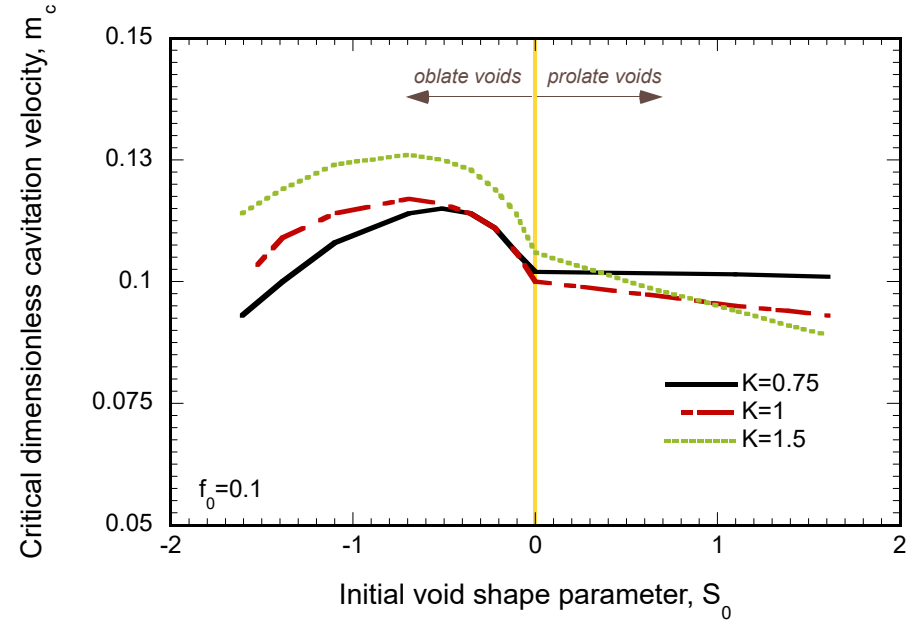

(b)

Figure 14: Results corresponding to three different values of the anisotropy parameter: $K=0.75, K=1$ (isotropic plasticity) and $K=1.5$. Variation in the critical dimensionless cavitation velocity $m_{c}$ with the initial void shape parameter $S_{0}$ for two different values of the initial void volume fraction: (a) $f_{0}=0.001$ and (b) $f_{0}=0.1$.

\section{Summary and concluding remarks}

In this work, we have obtained a self-similar solution for the steady-state expansion of a pressurized cylindrical cavity in an infinite porous medium described with the constitutive framework developed by Monchiet et al. (2008). The theoretical model is based on previous developments of Durban and co-workers (Durban and Masri, 2004; Masri and Durban, 2005; Cohen et al., 2010; Cohen and Durban, 2013) and includes the artificial viscosity approach of Lew et al. (2001) to capture the formation of plastic shocks at high cavity expansion velocities. We have carried out a comprehensive parametric analysis which encompasses calculations with different initial void shapes $-1.6 \leq S_{0} \leq 1.6$, a wide range of cavity expansion velocities $0.05 \leq m \leq 0.4$, and different anisotropy parameter values varying between $0.75 \leq K \leq 1.5$. The theoretical model predicts that the relationship between applied pressure and cavity expansion velocity is virtually independent of the shape of the voids included in the porous medium. In contrast, considering that the infinite porous medium contains initially spherical and prolate voids leads to important differences in the elastoplastic fields that develop near the cavity when compared to initially oblate voids. Specifically, spherical and prolate voids favor early formation of shock waves, leading to strong gradients in the radial profiles of stress and porosity. In addition, we have derived an analytical expression for the critical cavity expansion velocity leading to shock waves formation, $m_{c}$, which shows that for initially oblate voids the relationship between $m_{c}$ and $S_{0}$ is nonlinear, featuring a concave-downward shape with a maximum value of $m_{c}$ for an intermediate value of $S_{0}$, while for initially prolate voids the value of $m_{c}$ decreases roughly linearly 
with the increase of $S_{0}$. Furthermore, we have shown that the effect of the anisotropy parameter $K$ on the value of $m_{c}$ is such that increasing $K$ delays the formation of plastic shocks for initially oblate voids, while the opposite trend is generally obtained for initially prolate voids. These results are substantiated for different initial void volume fractions within the range $0.001 \leq f_{0} \leq 0.1$

All the theoretical results presented in this work have been compared with finite element simulations performed in Abaqus/Explicit using a VUMAT subroutine, in which the model of Monchiet et al. (2008) was implemented following the integration scheme developed by Hosseini and Rodríguez-Martínez (2021). The agreement between numerical simulations and theoretical model predictions for all the cavitation velocities and material features investigated in this work provides additional verification of the cavity expansion solution, supporting the results and conclusions presented in this paper (the comparison is not shown for the sake of brevity). However, validation of the theoretical predictions shown herein will be pending until relevant experimental results become available. Note also that the results presented in this paper are specific for the constitutive framework of Section 2 (which is based on specific evolution laws for the shape of the voids during deformation), in which the material porosity stands for the single damage parameter with no contribution of the voids size, so that the effect of microinertia -local acceleration fields that develop near the pores during dynamic compaction- on the mechanical response of the material is not accounted for (Czarnota et al., 2017, 2020). This is a potential limitation of the self-similar cavity expansion model that shall be further assessed using finite element simulations including the actual porous microstructure of the material (see the recent work of Marvi-Mashhadi et al. (2021)). Such calculations are part of a work in progress that will help to elucidate the specific role of voids size distribution on the structure of plastic shock waves in porous metallic materials.

\section{Funding}

The research leading to these results has received funding from the European Research Council (ERC) under the European Union's Horizon 2020 research and innovation programme. Project PURPOSE, grant agreement 758056 .

TdS wishes to acknowledge the support of FAPERGS, Fundação de Amparo à Pesquisa do Estado do Rio Grande do Sul, grant agreements 19/2551 - 0001054 - 0 and 21/2551 - $0000744-3$.

\section{Conflict of interest}

The authors declare that they have no conflict of interest. 


\section{Supplementary material}

In the following link we provide access to the MATLAB code with the implementation of the dynamic cavity expansion model: https://doi.org/10.21950/AZ6YF7.

\section{Author contributions}

T. dos Santos: Conceptualization; Formal analysis; Funding acquisition; Investigation; Software; Methodology; Project administration; Resources; Supervision; Validation; Visualization; Writing - original draft; Writing - review \& editing. J. C. Nieto-Fuentes: Formal analysis; Investigation; Validation; Writing - original draft; Writing - review \& editing. N. Hosseini: Formal analysis; Investigation; Software; Validation; Writing - review \& editing. J. A. Rodríguez-Martínez: Conceptualization; Formal analysis; Funding acquisition; Investigation; Methodology; Project administration; Resources; Supervision; Validation; Visualization; Writing - original draft; Writing - review \& editing.

\section{References}

Benzerga, A.A., Besson, J., 2001. Plastic potentials for anisotropic porous solids. European Journal of Mechanics - A/Solids 20, $397-434$.

Cohen, T., Durban, D., 2013. Hypervelocity cavity expansion in porous elastoplastic solids. Journal of Applied Mechanics 80, 011017.

Cohen, T., Masri, R., Durban, D., 2010. Shock waves in dynamic cavity expansion. Journal of Applied Mechanics $77,041009$.

Costas, M., Edwards-Mowforth, M., Kristoffersen, M., Teixeira-Dias, F., Brøtan, V., Paulsen, C.O., Børvik, T., 2021. Ballistic impact resistance of additive manufactured high-strength maraging steel: An experimental study. International Journal of Protective Structures , 20414196211035486.

Czarnota, C., Molinari, A., Mercier, S., 2017. The structure of steady shock waves in porous metals. Journal of the Mechanics and Physics of Solids 107, 204-228.

Czarnota, C., Molinari, A., Mercier, S., 2020. Steady shock waves in porous metals: Viscosity and micro-inertia effects. International Journal of Plasticity 135, 102816.

de Terris, T., Andreau, O., Peyre, P., Adamski, F., Koutiri, I., Gorny, C., Dupuy, C., 2019. Optimization and comparison of porosity rate measurement methods of selective laser melted metallic parts. Additive Manufacturing $28,802-813$. 
dos Santos, T., N'souglo, K.E., Rodríguez-Martínez, J.A., 2019a. Dynamic spherical cavity expansion in Gurson materials with uniform and non-uniform distributions of porosity. Mechanics of Materials 134, 115-131.

dos Santos, T., Vaz-Romero, A., Rodríguez-Martínez, J.A., 2019b. Dynamic cylindrical cavity expansion in orthotropic porous ductile materials. International Journal of Impact Engineering 132, 103325.

du Plessis, A., Yadroitsava, I., Yadroitsev, I., 2020. Effects of defects on mechanical properties in metal additive manufacturing: A review focusing on X-ray tomography insights. Materials \& Design 187, 108385.

Durban, D., Fleck, N.A., 1997. Spherical cavity expansion in a Drucker-Prager solid. Journal of Applied Mechanics $64,743-750$.

Durban, D., Masri, R., 2004. Dynamic spherical cavity expansion in a pressure sensitive elastoplastic medium. International Journal of Solids and Structures 41, 5697-5716.

Forrestal, M., Brar, N., Luk, V., 1991. Penetration of strain-hardening targets with rigid spherical-nose rods .

Forrestal, M., Luk, V., Brar, N., 1990. Perforation of aluminum armor plates with conical-nose projectiles. Mechanics of Materials 10, 97-105.

Forrestal, M.J., Warren, T.L., 2009. Perforation equations for conical and ogival nose rigid projectiles into aluminum target plates. International Journal of Impact Engineering 36, 220-225.

Gologanu, M., Leblond, J.B., Devaux, J., 1993. Approximate models for ductile metals containing non-spherical voids-case of axisymmetric prolate ellipsoidal cavities. Journal of the Mechanics and Physics of Solids 41, $1723-1754$.

Gologanu, M., Leblond, J.B., Devaux, J., 1994. Approximate models for ductile metals containing nonspherical voids-case of axisymmetric oblate ellipsoidal cavities. Journal of Engineering Materials and Technology 116, 290-297.

Gologanu, M., Leblond, J.B., Perrin, G., Devaux, J., 1997. Recent extensions of Gurson's model for porous ductile metals, in: Continuum micromechanics. Springer, pp. 61-130.

Gray, G., Livescu, V., Rigg, P., Trujillo, C., Cady, C., Chen, S., Carpenter, J., Lienert, T., Fensin, S., 2017. Structure/property (constitutive and spallation response) of additively manufactured 3161 stainless steel. Acta Materialia 138, 140 - 149.

Gurson, A., 1977. Continuum theory of ductile rupture by void nucleation and growth. Part I: Yield criteria and flow rules for porous ductile media. ASME Journal of Engineering Materials and Technology 99, 2-15. 
Hill, R., 1948. A theory of the yielding and plastic flow of anisotropic metals. Proceedings of the Royal Society of London. Series A, Mathematical and Physical Sciences 193, 281-297.

Hosseini, N., Rodríguez-Martínez, J.A., 2021. A simple and computationally efficient stress integration scheme based on numerical approximation of the yield function gradients: Application to advanced yield criteria. Finite Elements in Analysis and Design 192, 103538.

Keralavarma, S., Benzerga, A., 2010. A constitutive model for plastically anisotropic solids with non-spherical voids. Journal of the Mechanics and Physics of Solids 58, $874-901$.

Keralavarma, S., Benzerga, A., 2015. Numerical assessment of an anisotropic porous metal plasticity model. Mechanics of Materials 90, 212 - 228. Proceedings of the IUTAM Symposium on Micromechanics of Defects in Solids.

Kristoffersen, M., Costas, M., Koenis, T., Brøtan, V., Paulsen, C.O., Børvik, T., 2020. On the ballistic perforation resistance of additive manufactured alsi10mg aluminium plates. International Journal of Impact Engineering $137,103476$.

Kweon, S., Sagsoy, B., Benzerga, A., 2016. Constitutive relations and their time integration for anisotropic elasto-plastic porous materials. Computer Methods in Applied Mechanics and Engineering 310, 495 - 534.

Lew, A., Radovitzky, R., Ortiz, M., 2001. An artificial-viscosity method for the lagrangian analysis of shocks in solids with strength on unstructured, arbitrary-order tetrahedral meshes. Journal of Computer-Aided Materials Design 8, 213-231.

Madou, K., Leblond, J.B., 2012a. A Gurson-type criterion for porous ductile solids containing arbitrary ellipsoidal voids-I: Limit-analysis of some representative cell. Journal of the Mechanics and Physics of Solids 60, 1020-1036.

Madou, K., Leblond, J.B., 2012b. A Gurson-type criterion for porous ductile solids containing arbitrary ellipsoidal voids-II: Determination of yield criterion parameters. Journal of the Mechanics and Physics of Solids 60, 10371058.

Marvi-Mashhadi, M., Vaz-Romero, A., Sket, F., Rodríguez-Martínez, J.A., 2021. Finite element analysis to determine the role of porosity in dynamic localization and fragmentation: Application to porous microstructures obtained from additively manufactured materials. International Journal of Plasticity 143.

Masri, R., Durban, D., 2005. Dynamic spherical cavity expansion in an elastoplastic compressible Mises solid. Journal of Applied Mechanics 72, 887-898.

Masri, R., Durban, D., 2009. Deep penetration analysis with dynamic cylindrical cavitation fields. International Journal of Impact Engineering 36, $830-841$. 
Monchiet, V., Cazacu, O., Charkaluk, E., Kondo, D., 2008. Macroscopic yield criteria for plastic anisotropic materials containing spheroidal voids. International Journal of Plasticity 24, 1158 - 1189.

Morin, L., 2015. Influence des effets de forme et de taille des cavités, et de l'anisotropie plastique sur la rupture ductile. Ph.D. thesis. Université Pierre et Marie Curie-Paris VI.

Morin, L., Leblond, J.B., Kondo, D., 2015. A Gurson-type criterion for plastically anisotropic solids containing arbitrary ellipsoidal voids. International Journal of Solids and Structures 77, 86 - 101.

Sola, A., Nouri, A., 2019. Microstructural porosity in additive manufacturing: The formation and detection of pores in metal parts fabricated by powder bed fusion. Journal of Advanced Manufacturing and Processing 1, e10021.

Srivastava, A., Ponson, L., Osovski, S., Bouchaud, E., Tvergaard, V., Needleman, A., 2014. Effect of inclusion density on ductile fracture toughness and roughness. Journal of the Mechanics and Physics of Solids 63, 62-79.

Thompson, M.K., Moroni, G., Vaneker, T., Fadel, G., Campbell, R.I., Gibson, I., Bernard, A., Schulz, J., Graf, P., Ahuja, B., Martina, F., 2016. Design for additive manufacturing: Trends, opportunities, considerations, and constraints. CIRP Annals 65, 737-760.

Tvergaard, V., Nyvang Legarth, B., 2019. Effects of anisotropy and void shape on cavitation instabilities. International Journal of Mechanical Sciences 152, 81-87.

Wilkins, M.L., 1980. Use of artificial viscosity in multidimensional fluid dynamic calculations. Journal of Computational Physics 36, $281-303$. 